\title{
WIGNER COEFFICIENTS FOR THE PROTON-NEUTRON QUASISPIN GROUP: An application of vector coherent state techniques
}

\author{
K.T. HECHT' \\ Physics Department, University of Michigan, Ann Arbor, MI 48109, USA
}

Received 8 September 1988

\begin{abstract}
SO}(5) \supset \mathrm{U}(2)$ reduced Wigner coefficients, needed to extract the $n, T$-dependence of nuclear matrix elements in the seniority scheme, are evaluated by vector coherent state techniques by casting operators other than the group generators into their Bargmann $z$-space realizations. Results are given, in terms of simple angular momentum recoupling coefficients and the $K$-matrix elements of vector coherent state theory, for $\mathrm{SO}(5)$ couplings involving the 4-, 5-, and 10-dimensional representations. Both a simplification of earlier results and a generalization to states of arbitrarily high seniority has been achieved.
\end{abstract}

\section{Introduction}

In the past few years a generalized coherent state theory, termed vector coherent state theory ${ }^{1-4}$ ), and its associated $K$-matrix technique ${ }^{1,5}$ ) have been used to great advantage to give very explicit matrix representations of many of the higher rank symmetry algebras of interest in physical applictions. To date most of the detailed applications have focused on the matrix representations, that is, on the matrix elements of the generators of the algebras. [For a review and a more complete listing of recent applications see ref. $\left.{ }^{6}\right)$.] The vector coherent state technique, however, is in principle also a powerful tool for the detailed evaluation of the full Wigner-Racah calculus of the higher rank algebras. This has been illustrated for the elementary reduced-Wigner coefficients for $\mathrm{U}(n)$ which have been expressed ${ }^{7}$ ) in terms of multiplicity-free $\mathrm{U}(n-1)$ Racah coefficients and very simple $K$-matrix elements, the normalization factors of the vector coherent state theory. More recently, Le Blanc and Biedenharn ${ }^{8}$ ) have shown that some classes of $\mathrm{SU}(3) \supset \mathrm{SU}(2)$ reduced Wigner coefficients are simple products of $S U(2) 9-j$ coefficients and extremely simple SU(3) $K$-matrix ratios. It is not yet completely clear to what extent the spectacularly simple analytic form of such results can be generalized by means of vector coherent state theory to the case of the most general $\mathrm{SU}(3)$ couplings, particularly the cases involving outer multiplicities.

\footnotetext{
${ }^{1}$ Supported in part by the US National Science Foundation.
} 
For this reason it may be useful to derive general expressions for the needed Wigner coefficients of another simple example, with an $\mathrm{SU}(2)$ subgroup, the protonneutron quasispin group which is generated by an $\mathrm{SO}(5) \supset \mathrm{U}(2)$ algebra, (or its isomorphic $\mathrm{Sp}(4)$ algcbra). Matrix representations of this algebra have been discussed previously in terms of vector coherent state theory ${ }^{9,6}$ ). The development of the proton-neutron quasispin formalism into a useful tool for the nuclear spectroscopy of configurations of both protons and neutrons requires the explicit knowledge of many $\mathrm{SO}(5) \supset \mathrm{U}(2)$ reduced Wigner coefficients. With these the full $n, T$-dependence can be extracted from nuclear matrix elements in the scniority scheme ( $n=$ nucleon number, $T=$ isospin). Although very explicit expressions have been given previously ${ }^{10,11}$ ) for the $\mathrm{SO}(5)$ reduced Wigner coefficients of many of the low seniority representations of greatest interest in nuclear spectroscopy, a full analytic solution to this problem had been hampered by a "missing quantum number problem." Vector coherent state theory and the associated $K$-matrix constructions give an elegant solution to this problem in terms of the physically relevant coupling scheme in which a state of seniority $v$ and isospin $T$ is constructed by coupling the reduced isospin $t$ of the $v$ nucleons entirely free of $J=0$ coupled pairs with the resultant isospin $T_{n}$ of the $p$ pairs of nucleons coupled to $J=0, T=1$. The vector coherent state construction in terms of this coupling scheme was discussed in ref. ${ }^{9}$ ). It is the purpose of the present contribution to show how vector coherent state techniques can be used to calculate the $S O(5) \supset U(2)$ Wigner coefficients needed for nuclear spectroscopy. In particular, Wigner coefficients for the coupling of arbitrary irreducible representations with the 4-dimensional (spinor), 5-dimensional (vector) and 10-dimensional (regular) representations will be given in general analytic form. Both a simplification and a complete generalization of the earlier results ${ }^{10.11}$ ) has been achieved, making it possible to treat representations of arbitrarily high seniority.

The purpose of the present investigation is two-fold: One of the aims is a further refinement and completion of an elegant tool of nuclear spectroscopy. A second aim, however, involves the further development of the vector coherent state method in its application to the Wigner-Racah calculus of higher rank algebras. It is hoped that the techniques illustrated in some detail with the simple $\mathrm{SO}(5) \supset \mathrm{U}(2)$ algebra, involving mainly ordinary angular-momentum recoupling transformations, will also prove useful in more challenging symmetries.

\section{Vector coherent state realizations of the proton-neutron quasispin algebra}

Vector coherent state theory takes its simplest form for algebras with the following general structure: the generators of the algebra can be separated into a set of commuting raising operators, their hermitian-conjugate lowering operators, and a core subalgebra which contains the Cartan subalgebra of the full algebra. In its $S O(5) \supset U(2)$ version the proton-neutron quasispin algebra falls into this simple 
category. Since normalization and phase factors are vital for the evaluation of Wigner coefficients it will be important to give a careful definition of the various operators.

The raising operators are the $J=0, T=1$ pair creation operators defined by

$$
A^{\dagger}\left(M_{T}\right)=\frac{1}{2} \sum_{m} \sum_{m_{t_{1}}}(-1)^{j-m} a_{j m m_{t_{1}}}^{\vdots} a_{j-m m_{1},}^{\vdots}\left(\sum_{2}^{1} m_{t_{1}} \frac{1}{2} m_{t_{2}}\left|1 M_{T}\right\rangle\right. \text {. }
$$

The hermitian-conjugate lowering operators are the $J=0, T=1$ pair annihilation operators

$$
A\left(M_{T}\right)=\left(A^{*}\left(M_{T}\right)\right)^{\dagger} .
$$

The $\mathrm{U}(2)$ core subgroup is generated by the Cartan operator

$$
H_{1}=\frac{1}{2} N_{o p .}-\left(j+\frac{1}{2}\right)=\frac{1}{2} \sum_{m, m_{r}} a_{j m m_{1}}^{*} a_{j m m_{t}}-\left(j+\frac{1}{2}\right),
$$

and the isovector generators, $\boldsymbol{T}$, with standard spherical components

$$
T_{ \pm 1}=+\sqrt{\frac{1}{2}} \sum_{m} a_{j m \pm 1}^{*} a_{j m \neq \frac{1}{2}}, \quad T_{0}=\frac{1}{2} \sum_{m}\left(a_{j m+2}^{*} a_{j m+\frac{1}{2}}-a_{j m-\frac{1}{\dagger}}^{\dagger} a_{j m-!}\right) .
$$

(Note that a generalization to mixed configurations involving several $j$ subshells is immediate by including summations over both $j$ and $m$ and the replacement $\left(j+\frac{1}{2}\right) \rightarrow$ $\sum\left(j+\frac{1}{2}\right)$. Note also that $T_{0} \equiv H_{2}$.) It will also be useful to introduce cartesian components $A_{i}, A_{i}, T_{i},(i=1,2,3)$ defined in terms of the standard spherical components, e.g., by $A^{*}( \pm 1)=\mp \sqrt{\frac{1}{2}}\left(A_{1}^{*} \pm i A_{2}^{*}\right), A^{*}(0)=A_{3}^{*}$. SO(5) irreducible representations are to be labeled by the Cartan highest weights, $\left(\omega_{1} \omega_{2}\right)$,

$$
\omega_{1}=j+\frac{1}{2}-\frac{1}{2} v, \quad \omega_{2}=t,
$$

where $v$ and $t$ are seniority and reduced isospin, (the isospin of the $v$ nucleons entirely free of $J=0$ coupled pairs).

The single-nucleon creation and annihilation operators (for a fixed $j, m$ ) span the 4-dimensional irreducible representation ( $\left.\begin{array}{l}1 \\ 2 \\ 2\end{array}\right)$, while the 5-dimensional vector representation (10), and the 10-dimensional regular representation (11) are spanned by the bifermion operators coupled to odd $J\left(J_{\mathrm{o}}\right)$ and even $J\left(J_{\mathrm{e}}\right)$, respectively; where

$$
\begin{aligned}
& {\left[a^{*} \times a^{*}\right]_{M N_{r}}^{J T} \equiv \sum_{m_{1} m_{2}} \sum_{m_{t_{1}} m_{t_{2}}}\left\langle j m_{1} j m_{2} \mid J M\right\rangle\left\langle\frac{1}{2} m_{t_{1}} \frac{1}{2} m_{t_{2}} \mid T M_{T}\right\rangle a_{j m_{1} m_{t_{1}}}^{*} a_{m_{m_{2}} m_{t_{2}}}^{*}} \\
& {[a \times a]_{M M_{t}}^{\prime T} \equiv \sum_{m_{1} m_{2}} \sum_{m_{t_{1}} m_{t_{2}}}\left\langle j m_{1} j m_{2} \mid J M\right\rangle\left\langle\frac{1}{2} m_{t_{2}}^{1} m_{t_{2}} \mid T M_{T}\right\rangle} \\
& \times a_{j-m_{1}-m_{t_{1}}}(-1)^{j-m_{1}+\frac{1}{2}-m_{i}} a_{j-m_{2}-m_{t_{2}}}(-1)^{j-m_{2}+\frac{1}{1}-m_{t}} \\
& {\left[a^{*} \times a\right]_{M M_{1}}^{J T} \equiv \sum_{m_{1} m_{2}} \sum_{m_{t_{1}} m_{t_{2}}}\left\langle j m_{1} j m_{2} \mid J M\right\rangle\left\langle{ }_{2}^{1} m_{t_{1}} \frac{1}{2} m_{t_{2}} \mid T M_{T}\right\rangle} \\
& \times a_{j m_{1} m_{1}}^{\dagger} a_{j-m_{2}-m_{r_{2}}}(-1)^{j-m_{2}+\frac{1}{2}-m_{t_{2}}} .
\end{aligned}
$$

The relationship among standard $\mathrm{SO}(5)$ irreducible operators, $T_{H_{1} T M_{T}}^{\left(\omega_{1} \omega_{2}\right)}$, can be given through the matrix elements of the generators, e.g.

$$
\begin{aligned}
& {\left[A^{*}\left(M_{T_{0}}\right), T_{H_{1}, T M_{T}}^{\left(\omega_{i}{ }^{\prime}\right.}\right]} \\
& =\sum_{T^{\prime}} T_{H_{1}+1}^{\left(\omega_{1} t\right)} T^{\prime} M_{T}+M_{r_{1}}\left\langle\left(\omega_{1} t\right) H_{1}+1 T^{\prime} M_{T}+M_{T_{0}}\left|A^{\dagger}\left(M_{T_{0}}\right)\right|\left(\omega_{1} t\right) H_{1} T M_{T^{\prime}}\right\rangle .
\end{aligned}
$$


Standard $\mathrm{SO}(5)$ tensors $T^{\left(\frac{1}{2}\right)}, T^{(10)}, T^{(11)}$ are given explicitly in table 1. (Henceforth $J_{\mathrm{e}}$ will denote even $J$-values, with $J_{\mathrm{e}} \neq 0$, to distinguish such tensors from the $\mathrm{SO}(5)$ generators.)

The generalized vacuum states of coherent state thcory will be chosen as the $\mathrm{SO}(5)$ lowest weight states with $n=v$ nucleons which are annihilated by the $J=0$, $T=1$ pair annihilation operators

$$
A\left(M_{T}\right)\left|\omega_{1} t m_{t}\right\rangle=0 \quad \text { for all } m_{t}, M_{T} .
$$

In terms of this vector vacuum or so-called intrinsic state the generalized coherent state is defined by

$$
\left|z, \omega_{1} t m_{t}\right\rangle=\exp \left(z^{*} \cdot \boldsymbol{A}^{\dagger}\right)\left|\omega_{1} t m_{t}\right\rangle
$$

in terms of the three complex variables $z=\left(z_{1}, z_{2}, z_{3}\right)$, which can also be transcribed to standard spherical component form $z_{ \pm 1}=\mp \sqrt{\frac{1}{2}}\left(z_{1}+i z_{2}\right), z_{0}=z_{3}$.

State vectors $|\Psi\rangle$ are to be mapped into their $z$-space functional realizations

$$
|\Psi\rangle \rightarrow \Psi_{\omega_{1} t m_{t}}(\boldsymbol{z})=\left\langle\omega_{1} t m_{t}|\exp (\boldsymbol{z} \cdot \boldsymbol{A})| \Psi\right\rangle
$$

and operators $\mathscr{O}$ are mapped into their $z$-space realizations $\Gamma(\mathscr{O})$

$$
\begin{aligned}
\mathcal{O}|\Psi\rangle & \rightarrow \Gamma(\mathcal{O}) \Psi_{\omega_{1} t m_{1}}(z)=\left\langle\omega_{1} t m_{t}\left|\mathrm{e}^{(z \cdot A)} \mathcal{O}\right| \Psi\right\rangle \\
& =\left\langle\omega_{1} t m_{t}\left|\left\{\mathcal{O}+[(z \cdot \boldsymbol{A}), \mathcal{O}]+\frac{1}{2}[(z \cdot \boldsymbol{A}),[(z \cdot \boldsymbol{A}), \mathcal{O}]]+\cdots\right\} \mathrm{e}^{(z \cdot \boldsymbol{A})}\right| \Psi\right\rangle .
\end{aligned}
$$

The coherent state realization of the generators of the algebra were given in ref. ${ }^{9}$ )

\begin{tabular}{|c|c|c|}
\hline$H_{1}$ & $T$ & $\left(\frac{1}{2} \frac{1}{2}\right)$ operators \\
\hline $\begin{array}{l}+\frac{1}{2} \\
-\frac{1}{2}\end{array}$ & $\begin{array}{l}\frac{1}{2} \\
\frac{1}{2}\end{array}$ & 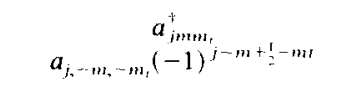 \\
\hline$H_{1}$ & $T$ & (10) operators \\
\hline $\begin{array}{r}+1 \\
0 \\
-1\end{array}$ & $\begin{array}{l}0 \\
1 \\
0\end{array}$ & $\begin{array}{l}\sqrt{\frac{1}{2}}\left[a^{+} \times a^{\dagger}\right]_{M 0}^{\prime} \\
{\left[a_{M}^{\prime} \times a\right]_{M}^{J} M_{M}^{\prime}} \\
\sqrt{\frac{1}{2}}[a \times a]_{M 0}^{M_{0}}\end{array}$ \\
\hline$H_{1}$ & $T$ & (11) operators \\
\hline $\begin{array}{r}+1 \\
0 \\
-1\end{array}$ & $\begin{array}{c}1 \\
0 ; 1 \\
1\end{array}$ & 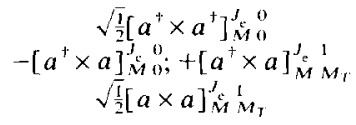 \\
\hline
\end{tabular}

$$
\Gamma(\boldsymbol{A})=\frac{\partial}{\partial \boldsymbol{z}} \equiv \boldsymbol{\nabla},
$$

TABLE 1

Basic tensor operators, $T_{i 1_{1} T_{M_{2}}}^{\left(\omega_{1} \omega_{2}\right)}$ 


$$
\begin{aligned}
\Gamma\left(H_{1}\right) & =-\omega_{1}+(z \cdot \nabla), \\
\Gamma(\boldsymbol{T}) & =\mathbb{t}-i[z \times \nabla], \\
\Gamma\left(\boldsymbol{A}^{\dagger}\right) & =\omega_{1} z-i[z \times \mathbb{Z}]-z(z \cdot \nabla)+\frac{1}{2}(z \cdot z) \nabla,
\end{aligned}
$$

where these operators are made up of "collective" or "orbital" parts which are functions of $z$ and $\nabla$, and "intrinsic" operators such as the three components of $t$ which commute with $z$ and $\nabla$ and need to be defined only through their action on the vacuum or intrinsic states $\left|\omega_{1} t m_{t}\right\rangle$, with $n=v$. Note from the commutator expansion of eq. (8) that these are to be understood from their left action on the vacuum states. Note also that $\mathbb{t}_{1}$, the intrinsic part of $H_{1}$, can be given through its eigenvalue, $-\omega_{1}$.

Although it is possible to define a $z$-space scalar product with measure such that the $z$-space operators $\Gamma\left(\boldsymbol{A}^{*}\right)$ are adjoints of $\Gamma(\boldsymbol{A})$, (this would be the conventional coherent state theory procedure), it is advantageous to define the $z$-space scalar product in terms of complex $z$-plane integrations with the standard Bargmann measure, $\pi^{-3} \exp \left[-\left(z \cdot z^{*}\right)\right]$, in this case involving a 3 -dimensional $z$. With this measure $\nabla_{i}$ is the adjoint of $z_{i}$; and the $\operatorname{SO}(5)$ algebra has been mapped into a direct sum of a three-dimensional oscillator algebra generated by the $z_{i}, \nabla_{i}$ and an intrinsic $U(1) \times S U(2)$ algebra generated by the intrinsic operators $b_{1}$, and t. The price paid for this simple structure is that the realization, (9), is a nonunitary or Dyson realization of the SO(5) algebra. The transformation to a unitary (HolsteinPrimakoff) realization is then made via a similarity transformation with the operator $K$

$$
\gamma\left(\boldsymbol{A}^{\dagger}\right)=K^{-1} \Gamma\left(\boldsymbol{A}^{\dagger}\right) K, \quad \gamma(\boldsymbol{A})=K^{-1} \Gamma(\boldsymbol{A}) K .
$$

Since the $\Gamma\left(H_{1}\right), \Gamma(T)$ form a unitary realization of the U(2) subgroup, $K$ can be chosen to be a number-conserving, $\mathrm{SU}(2)$-invariant operator which commutes with $\Gamma\left(H_{1}\right), \Gamma(\boldsymbol{T})$; and the matrix elements of $K$ will therefore be diagonal in $n$ and $T$ and independent of $M_{T}$. The unitary requirement $\gamma\left(\boldsymbol{A}^{\dagger}\right)=(\gamma(\boldsymbol{A}))^{\dagger}$, together with eq. $(9 \mathrm{a})$, and $\left(\nabla_{i}\right)^{\dagger}=z_{i}$ leads to

$$
\Gamma\left(A^{\dagger}\right) K K^{\dagger}=K K^{\dagger} z .
$$

This equation can be solved for the needed $K K^{\prime \prime}$ most easily by the introduction of the auxiliary operator, $\Lambda_{\text {op }}$, (the "Toronto trick"), where

$$
\left[\Lambda_{\mathrm{op}}, z\right]=\Gamma\left(\boldsymbol{A}^{*}\right) .
$$

It is straightforward to show that this relation is satisfied by the operator

$$
A_{\mathrm{op}}=\frac{1}{2}(z \cdot \nabla)(z \cdot \nabla)+\frac{1}{4}(z \cdot z) \nabla^{2}+\left(\omega_{1}+\frac{1}{2}\right)(z \cdot \nabla)+i(t \cdot\lfloor z \times \nabla]) .
$$


Specific examples of the matrix elements of the hermitian operator $\left(K K^{\dagger}\right)$ were given in ref. ${ }^{9}$ ). A more complete tabulation of analytic formulae will be given in an appendix, and a numerical evaluation for even more challenging cases is straightforward with recursion formulac which follow from eqs. (11)-(13); see appendix A. For many of the irreducible representations of $\mathrm{SO}(5)$, as for many of the other applications of vector coherent state theory, it is possible to make $K$ itself hermitian. With $K=K^{\dagger},\left(K K^{\dagger}\right)$ can be renamed $K^{2}$. [This notation was used throughout refs. $\left.{ }^{6,9}\right)$.] Since cases where $\left(K K^{\dagger}\right)$ has zero eigenvalues must be included, the assumption $K=K^{\dagger}$ will not be made $a b$ initio and will be introduced only in the very end of a calculation in those cases in which it is valid.

By means of the coherent state approach the $\mathrm{SO}(5)$ algebra has been mapped into a simpler algebra, a direct sum of a 3-dimensional oscillator algebra and an intrinsic $U(2)$ algebra. The basis vectors of an $\mathrm{SO}(5)$ irreducible representation in the $z$-space functional realization can then be given by the vector coupled state

$$
\begin{aligned}
\left.\mid p \omega_{1}\left[t \times T_{p}\right] T M_{T}\right) & \left.=\left[Z_{T_{p}}^{(p)}(z) \times \mid \omega_{1} t\right)\right]_{T M_{r}} \\
& \left.=\sum_{m, M_{T^{\prime}}} Z_{T_{p}, M_{i_{p}}}^{(p 0)}(z) \mid \omega_{1} t m_{t}\right)\left\langle t m_{t} T_{p} M_{T_{p}} \mid T M_{T}\right\rangle,
\end{aligned}
$$

where the $Z^{(p 0)}(z)$ is a normalized $z$-space 3 -dimensional harmonic-oscillator function

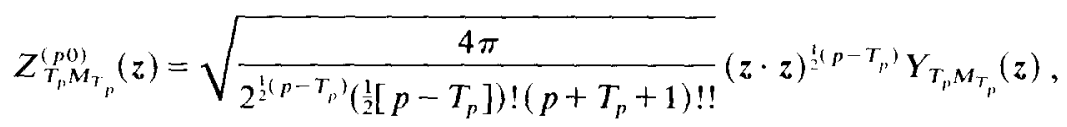

the Bargmann space version of a Moshinsky $S U(3) \supset S O(3)$ oscillator function transforming according to the totally symmetric $\operatorname{SU}(3)$ representation $(p 0)$ with angular momentum $T_{p}$, with the well-known restriction $T_{p}=p, p-2, \ldots, 0$ (or 1 ). In eq. (15), $Y$ is a standard solid harmonic in $z$. The quantum number $p$ measures the number of $J=0$-coupled pairs, $p=\frac{1}{2}(n-v)$. (Alternately, $\left.p=\left(H_{1}\right)_{\text {eigen }}+\omega_{1}\right)$. The state construction thus proceeds via standard angular momentum coupling of the reduced isospin $t$ of the $v$ nucleons free of $J=0$-coupled pairs with the resultant isospin $T_{p}$ of the $p$ pairs of nucleons coupled to $J=0, T=1$. Note in particular that the coupling order is $\left[t \times T_{p}\right]$, i.e. a right to left coupling order is implied by the large square bracket of eq. (14). All subsequent equations will adhere to this right to left coupling order convention in order to simplify angular momentum phase factors. (Note, however, that the standard operators of eqs. (1a) and (3) have been defined in terms of the conventional left to right coupling order. These will be the only equations of the paper which will not have used a right to left coupling order.)

The process of unitarization via the $K$ operator will introduce states which are linear combinations of states with different $T_{p}$ for a given $p\left(\omega_{1} t\right) T M_{T}$. Such states are to be designated by quantum numbers $i$ or $i^{\prime}$. From eqs. (10) and (11) the unitary 
$z$-space operator can be put in the form

$$
\gamma\left(\boldsymbol{A}^{\dagger}\right)=K^{\dagger} \boldsymbol{z}\left(K^{\dagger}\right)^{-1}
$$

leading to the angular momentum reduced matrix element

$$
\begin{aligned}
& \left(n+2\left(\omega_{1} t\right) T^{\prime} i^{\prime}\left\|\gamma\left(\boldsymbol{A}^{\dagger}\right)\right\| n\left(\omega_{1} t\right) T i\right) \\
& \quad=\sum_{T_{p}^{\prime} T_{p}}\left(K^{\dagger}\right)_{i^{\prime} T_{p}^{\prime}}\left(p+1 \omega_{1}\left[t \times T_{p}^{\prime}\right] T^{\prime}\|z\| p \omega_{1}\left[t \times T_{p}\right] T\right)\left(\left(K^{\dagger}\right)^{-1}\right)_{T_{p^{\prime}}} \\
& \quad=\left\langle n+2\left(\omega_{1} t\right) T^{\prime} i^{\prime}\left\|\boldsymbol{A}^{\dagger}\right\| n\left(\omega_{1} t\right) T i\right\rangle .
\end{aligned}
$$

The reduced matrix element of the operator $z$ is given by standard angular momentum coupling theory

$$
\begin{aligned}
\left(p+1 \omega_{1}\left[t \times T_{p}^{\prime}\right] T^{\prime}\|z\| p \omega_{1}\left[t \times T_{p}\right] T\right) & =\left[\begin{array}{ccc}
t & T_{p} & T \\
0 & 1 & 1 \\
t & T_{p}^{\prime} & T^{\prime}
\end{array}\right]\left(T_{p}^{\prime}\|z\| T_{p}\right) \\
& =U\left(t T_{p} T^{\prime} 1 ; T T_{p}^{\prime}\right)\left(T_{p}^{\prime}\|z\| T_{p}\right),
\end{aligned}
$$

where the 3-dimensional oscillator reduced matrix element is given in terms of a simple $\mathrm{SU}(3) \supset \mathrm{SO}(3)$ reduced Wigner coefficient

$$
\left(T_{p}^{\prime}\|z\| T_{p}\right)=\sqrt{p+1}\left\langle(p 0) T_{p} ;(10) 1 \|(p+1,0) T_{p}^{\prime}\right\rangle .
$$

The very few simple $\mathrm{SU}(3) \supset \mathrm{SO}(3)$ Wigner coefficients of this type needed in this investigation are collected in table 2 . Since we have used the unitary $z$-space realization $\gamma\left(\boldsymbol{A}^{\prime}\right)$ of $\boldsymbol{A}^{\prime}$ in an orthonormal basis, the result is representation-independent and can be transformed to the standard Hilbert space basis, as indicated in

TABLE 2

Needed SU(3) Wigner coefficients

$$
\begin{aligned}
& \left\langle(p 0) T_{p} ;(10) 1 \|(p+1,0) T_{p}+1\right\rangle=\sqrt{\frac{\left(T_{p}+1\right)\left(p+T_{p}+3\right)}{\left(2 T_{p}+3\right)(p+1)}} \\
& \left\langle(p 0) T_{p} ;(10) 1 \|(p+1,0) T_{p}-1\right\rangle=-\sqrt{\frac{T_{p}\left(p-T_{p}+2\right)}{\left(2 T_{p}-1\right)(p+1)}} \\
& \left\langle(p 0) T_{p} ;(20) 2 \|(p+2,0) T_{p}+2\right\rangle=\sqrt{\frac{\left(T_{p}+1\right)\left(T_{p}+2\right)\left(p+T_{p}+3\right)\left(p+T_{p}+5\right)}{\left(2 T_{p}+3\right)\left(2 T_{p}+5\right)(p+1)(p+2)}} \\
& \left\langle(p 0) T_{p} ;(20) 2 \|(p+2,0) T_{p}-2\right\rangle-\sqrt{\frac{T_{p}\left(T_{p}-1\right)\left(p-T_{p}+2\right)\left(p-T_{p}+4\right)}{\left(2 T_{p}-1\right)\left(2 T_{p}-3\right)(p+1)(p+2)}} \\
& \left\langle(p 0) T_{p} ;(20) 2 \|(p+2,0) T_{p}\right\rangle=-\sqrt{\frac{2 T_{p}\left(T_{p}+1\right)\left(p+T_{p}+3\right)\left(p-T_{p}+2\right)}{3\left(2 T_{p}-1\right)\left(2 T_{p}+3\right)(p+1)(p+2)}} \\
& \left\langle(p 0) T_{p} ;(20) 0 \|(p+2,0) T_{p}\right\rangle=\sqrt{\frac{\left(p+T_{p}+3\right)\left(p-T_{p}+2\right)}{3(p+1)(p+2)}}
\end{aligned}
$$


the last line of eq. (17). A further remark about the notation: State vectors are of course defined independent of their representation so that the standard $|\ldots\rangle$ symbol could have been used in all steps of eq. (17). The presence of a $\gamma$ (or $\Gamma$ ) symbol, or the explicit appearance of functions of $z, \nabla$, and intrinsic (double linc) operators should automatically signal that matrix elements should be interpreted through their $z$-space integrations. Since we shall switch from z-space to standard Hilbert space form in many equations, state vectors have in addition been designated by $\mid .$. ) to signal more specifically that $z$-space realizations are implied, whereas standard ket symbols $|\ldots\rangle$ are to imply standard realizations. Finally, angular momentum reduced matrix elements are defined without $[2 T+1]^{1 / 2}$ dimensional factors, so that

$$
\left\langle\alpha^{\prime} T^{\prime}\left\|\boldsymbol{A}_{1}^{\dagger}\right\| \alpha T\right\rangle=\left\langle\alpha^{\prime} T^{\prime} M_{T}^{\prime}\right|\left[\boldsymbol{A}_{1}^{\dagger} \times|\alpha T\rangle\right]_{T^{\prime} M_{j}^{\prime}},
$$

and

$$
\left\langle\alpha T\|\boldsymbol{A}\| \alpha^{\prime} T^{\prime}\right\rangle=\sqrt{\frac{2 T^{\prime}+1}{2 T+1}}(-1)^{T-T^{\prime}}\left\langle\alpha^{\prime} T^{\prime}\left\|\boldsymbol{A}^{\dagger}\right\| \alpha T\right\rangle .
$$

Since all matrices $\left(K K^{\dagger}\right)_{T_{p}^{\prime} T_{n}}$ of this investigation will be purely real, the notation will be simplified via

$$
\left(K^{\dagger}\right)_{i T_{p}}=(K)_{T_{p}, i}, \quad\left(\left(K^{+}\right)^{-1}\right)_{T_{p},}=\left(K^{-1}\right)_{i T_{p}} .
$$

The process of finding the $K$ and $K^{-1}$ matrix elements involves the diagonalization of the real hermitian matrices $\left(K K^{\dagger}\right)$ via a unitary matrix

$$
\left(K K^{\dagger}\right)=U^{\dagger} \lambda U
$$

where $\lambda$ is a real positive semidefinite diagonal matrix, $\lambda_{i j}=\lambda_{i} \delta_{i j}$. Zero eigenvalues of $\lambda$ immediately signal the occurrence of Pauli-forbidden states. If $\lambda_{i}$ denotes a nonzero eigenvalue, eq. (23) can be solved for $K$, yielding

$$
K_{T_{p} i}=\left(U^{\dagger}\right)_{T_{p}, i} \sqrt{\lambda_{i}}, \quad\left(K^{-1}\right)_{i T_{p}}=\frac{1}{\sqrt{\lambda_{i}}} U_{i T_{r}} .
$$

In refs. $\left.{ }^{6,9}\right)$ it was shown that the $\left(K K^{*}\right)$ matrices are often nearly diagonal, particularly in the most important cases of relatively high $\left(j+\frac{1}{2}\right)$ values. The labels, $i$, were therefore replaced by the numerical value of the dominant $T_{p}$ in the $i$ th state. In the present investigation the distinction between the two types of labels will be carefully preserved. For many $S O(5)$ irreducible representations, and for many special states within arbitrary representations the $\left(K K^{\dagger}\right)$ matrices will be 1-dimensional corresponding to the fact that $T_{p}$ is uniquely specified by $p$ and $T$. In such cases, $K=K^{\dagger}$, and $i$ can be replaced by $T_{p}$. In such cases eq. (11) leads to a simple recursion relation for $\left(K K^{\dagger}\right)=K^{2}$. Substituting $\left[\Lambda_{\text {op }}, z\right]$ for $\Gamma\left(\boldsymbol{A}^{\dagger}\right)$, and taking matrix elements between states $\left.\mid p \omega_{1}\left[t \times T_{p}\right] T\right)$ and $\left(p+1 \omega_{1}\left[t \times T_{p}^{\prime}\right] T^{\prime} \mid\right.$ of 
eq. (11) in cases with both uniquely specified $T_{p}$, and $T_{p}^{\prime}$, this yields

$$
\frac{\left(K^{2}\left(p+1\left(\omega_{1} t\right) T^{\prime}\right)\right)_{T_{p}^{\prime} T_{p^{\prime}}^{\prime}}}{\left(K^{2}\left(p\left(\omega_{1} t\right) T\right)\right)_{T_{p} T_{p}}}=A_{p+1 T_{p}^{\prime} T^{\prime}}-A_{p T_{p} T} .
$$

The eigenvalues of $A_{\text {op }}$ follow at once from eqs. (13)-(15),

$$
A_{p T_{p} T}=-\frac{1}{4} p(p-1)+p\left(\omega_{1}+\frac{1}{2}\right)-\frac{1}{2} T(T+1)+\frac{1}{2} t(t+1)+{ }_{4}^{1} T_{p}\left(T_{p}+1\right) .
$$

The states with $p=1$ will play a special role in this investigation. Since $T_{p}=1$ only, the $K^{2}$ matrices for these states will be 1-dimensional in all representations $\left(\omega_{1} t\right)$. Since the intrinsic states $\left.\mid \omega_{1} t m_{t}\right)$ will be assumed to be normalized so that $K=1$ for $p=0$, eqs. (25) and (26) yield

$$
\begin{aligned}
\left(K^{2}\left(1\left(\omega_{1} t\right) T\right)\right)_{11} & =\omega_{1}+1-\frac{1}{2} T(T+1)+\frac{1}{2} t(t+1) \\
& = \begin{cases}\omega_{1}-t & \text { for } T=t+1 \\
\omega_{1}+1 & \text { for } T=t \\
\omega_{1}+t+1 & \text { for } T=t-1 .\end{cases}
\end{aligned}
$$

A full discussion of the evaluation of the $\left(K K^{+}\right)$matrices will be given in appendix A. As a final remark, it will also be useful to recall the role of the $\left(K K^{\dagger}\right)$ matrices as overlap matrices. Inverting eq. (16), a polynomial in $z$ can be converted into a polynomial in $\boldsymbol{A}^{\dagger}$ via

$$
z \times z \times \cdots \times z=\left(K^{\dagger}\right)^{-1} \gamma\left(\boldsymbol{A}^{\dagger}\right) K^{\dagger}\left(K^{\dagger}\right)^{-1} \gamma\left(\boldsymbol{A}^{\dagger}\right) K^{\dagger} \cdots\left(\boldsymbol{K}^{\dagger}\right)^{-1} \gamma\left(\boldsymbol{A}^{\dagger}\right) K^{\dagger} .
$$

Combination of interior $K^{\dagger}\left(K^{\dagger}\right)^{-1}$ factors and the starting value $K_{p=0}^{\dagger}=1$ leads to a conversion of the orthonormal $z$-space basis into an orthonormal basis in standard representation.

$$
\begin{aligned}
{\left.\left[Z_{T_{r}}^{\left(p^{0}\right)}(z) \times \mid \omega_{1} t\right)\right]_{T M_{T}} } & \left.\Rightarrow \sum_{T_{p}}\left(K^{-1}\right)_{i T_{p}}\left[Z_{T_{p}}^{(p)}\left(\boldsymbol{A}^{\dagger}\right) \times \mid \omega_{1} t\right)\right]_{T M_{T}} \\
& =\left|p\left(\omega_{1} t\right) T M_{T} i\right\rangle .
\end{aligned}
$$

If we we define the nonorthonormal state vectors

$$
\left[Z_{T_{p}}^{(p)}\left(\boldsymbol{A}^{\dagger}\right) \times\left|\omega_{1} t\right\rangle\right]_{T_{M_{T}}} \equiv\left|\Psi\left(p \omega_{1}\left[t \times T_{p}\right] T M_{T}\right)\right\rangle,
$$

where $z$ has been replaced with the operator $\boldsymbol{A}^{\dagger}$ within $Z(z)$, the orthonormality of the $\left|p\left(\omega_{1} t\right) T M_{T} i\right\rangle$ leads to the overlap matrix

$$
\left\langle\Psi\left(p \omega_{1}\left[t \times T_{p}^{\prime}\right] T M_{T}\right) \mid \Psi\left(p \omega_{1}\left[t \times T_{p}\right] T M_{T}\right)\right\rangle=\left(K K^{\dagger}\right)_{T_{p} T_{n}} .
$$

\section{Calculation of $S O(5)$ Wigner coefficients}

To evaluate $\mathrm{SO}(5) \supset \mathrm{U}(2)$ reduced Wigner coefficients by vector coherent state techniques, it will be useful to construct the $z$-space realization of $S O(5)$ irreducible tensor operators, which are given in table 1 in terms of the nucleon creation and 
annihilation operators for some of the simpler irreducible representations. Straightforward commutator algebra and the application of eq. (8) yield

$$
\begin{aligned}
& \Gamma\left(a_{j m m_{t}}\right)=\mathbb{a}_{j m m_{t}}, \\
& \Gamma\left(a_{j m m_{t}}^{\dagger}\right)=\mathbb{a}_{j m m_{l}}^{\dagger}+\sqrt{\frac{3}{2}}\left[z^{1} \times \mathbb{Q}_{j m}^{1 / 2}\right]_{m_{1}}^{1 / 2},
\end{aligned}
$$

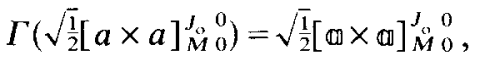

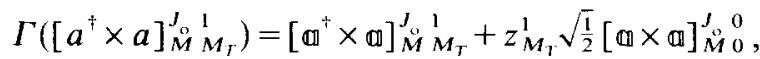

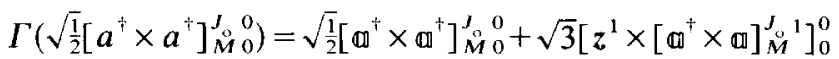

$$
\begin{aligned}
& +\sqrt{\frac{3}{2}} Z_{0}^{(20)}(z) \times \sqrt{\frac{1}{2}}[\mathbb{\infty} \times \mathbb{0}]_{M 0}^{J_{M} 0},
\end{aligned}
$$

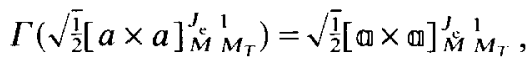

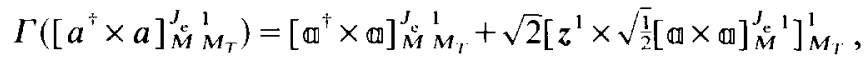

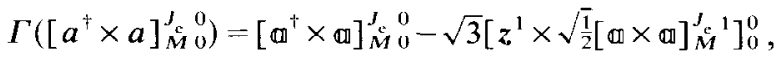

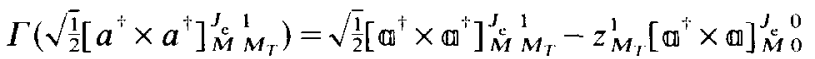

$$
\begin{aligned}
& +\sqrt{2}\left[z^{1} \times\left[\mathbb{\omega}^{\dagger} \times \mathbb{a}\right]_{M}^{J_{\dot{s}}^{1}}\right]_{M_{T}}^{1} \\
& +\sqrt{\frac{10}{3}}\left[Z_{2}^{(20)}(z) \times \sqrt{\frac{1}{2}}[\varpi \times \llbracket]_{M}^{s_{1}^{1}}\right]_{M_{T}}^{1} \\
& -\sqrt{\frac{1}{6}} Z_{0}^{(20)}(z) \times \sqrt{\frac{1}{2}}[\propto \times \mathbb{0}]_{M}^{J_{M}{ }^{\prime}{ }_{T}} \text {, }
\end{aligned}
$$

where, e.g.,

$$
\left[z^{1} \times \mathbb{a}_{j m}^{1 / 2}\right]_{m_{i}}^{1 / 2} \equiv \sum_{M_{T} m_{t}^{\prime}} \mathbb{Q}_{j-m-m_{t}^{\prime}}(-1)^{j-m+\frac{1}{2}-m_{i}^{\prime}} z_{M_{T}}^{1}\left\langle\frac{1}{2} m_{t}^{\prime} 1 M_{T} \mid \frac{1}{2} m_{t}\right\rangle .
$$

Finally, these nonunitary $z$-space realizations of the $\mathrm{SO}(5)$ irreducible tensor operators must be transformed to unitary form via the $K$ operation

$$
\gamma(a)=K^{-1} \Gamma(a) K, \quad \gamma\left(a^{\dagger}\right)=K^{-1} \Gamma\left(a^{\dagger}\right) K, \text { etc } .
$$

The double-lined operators $₫$ and $\varpi^{\dagger}$ are again intrinsic operators defined only through their action on the "vacuum" or intrinsic states, with $n=v$ only. These double-lined operators commute with $z$ and $\nabla$. From the defining relations for $\Gamma(\mathscr{O})$ of eq. (8), it is clear that these operators must be understood in terms of their left actions. Thus, the left action of $\mathbb{\varpi}^{\dagger}$, must convert the state with $n=v$ in $\left(\omega_{1} t m_{1} \mid \mathbb{\Phi}^{\dagger}\right.$ to a state with $n=v-1$ or with $\omega_{1}^{\prime}=\omega_{1}+\frac{1}{2}$. Since the intrinsic operators change $\mathrm{SO}(5)$ irreducible representations the first step of any calculation involves the evaluation of the reduced matrix elements of such operators. It will be convenient to write states with $n=v$ through a full set of quantum numbers $\left|j^{\mathrm{v}} t m_{t} \alpha J M\right\rangle$ or $\left|\omega_{1} t m_{t} \alpha J M\right\rangle$ where $\alpha$ is a shorthand for the additional $\mathrm{Sp}(2 j+1)$ quantum numbers needed for the full specification of the state with $n=v$ in the seniority scheme. None of the present $\mathrm{SO}(5)$ results, however, will depend on such quantum numbers. Defining matrix elements reduced with respect to both $J$ and $T$ [see eq. (20)], we have the obvious result mentioned above

$$
\left(\left(\omega_{1}+\frac{1}{2} t^{\prime}\right) \alpha^{\prime} J^{\prime}\left\|\mathbb{\varpi}^{\dagger}\right\|(\omega t) \alpha J\right)=0 .
$$


The case with $v^{\prime}=v+1$, or $\omega_{1}^{\prime}=\omega_{1}-\frac{1}{2}$, will be used to illustrate a very simple, yet nontrivial, case. In this case

$$
\begin{aligned}
\left\langle\left(\omega_{1}-\frac{1}{2} t^{\prime}\right) \alpha^{\prime} J^{\prime}\left\|a^{\dagger}\right\|\left(\omega_{1} t\right) \alpha J\right\rangle= & \left(\left(\omega_{1}-\frac{1}{2} t^{\prime}\right) \alpha^{\prime} J^{\prime}\left\|\gamma\left(a^{\dagger}\right)\right\|\left(\omega_{1} t\right) \alpha J\right) \\
= & \left(\left(\omega_{1}-\frac{1}{2} t^{\prime}\right) \alpha^{\prime} J^{\prime}\left\|K^{-1} \Gamma\left(a^{\dagger}\right) K\right\|\left(\omega_{1} t\right) \alpha J\right) \\
= & \left(\left(\omega_{1}-\frac{1}{2} t^{\prime}\right) \alpha^{\prime} J^{\prime}\left\|\mathbb{\Phi}^{\dagger}\right\|\left(\omega_{1} t\right) \alpha J\right) \\
& +\sqrt{\frac{3}{2}}\left(\left(\omega_{1}-\frac{1}{2} t^{\prime}\right) \alpha^{\prime} J^{\prime}\left\|\left[z^{1} \times \mathbb{D}^{1 / 2}\right]^{1 / 2}\right\|\left(\omega_{1} t\right) \alpha J\right) .
\end{aligned}
$$

(Note that the action of $K$ or $K^{-1}$ on intrinsic states is the simple unit operation.) The second term can be rewritten

$$
\begin{aligned}
& -\sqrt{\frac{3}{2}}\left(\omega_{1}-\frac{1}{2} t^{\prime} m_{t}^{\prime} \alpha^{\prime} J^{\prime} M^{\prime} \mid\left[\left[\Phi^{1 / 2} \times z^{1}\right]^{1 / 2} \times \mid\left(\omega_{1} t\right) \alpha J\right)\right]_{M^{\prime} m_{i}^{\prime}}^{J^{\prime} t^{\prime}} \\
& \quad=-\sqrt{\frac{3}{2}} \sum_{t^{\prime \prime}} U\left(t 1 t^{\prime \prime} ; t^{\prime \prime} \frac{1}{2}\right)\left(\left(\omega_{1}-\frac{1}{2} t^{\prime}\right) \alpha^{\prime} J^{\prime}\|\bowtie\|\left[z^{1} \times \mid\left(\omega_{1} t\right) \alpha J\right)\right]^{t^{\prime \prime}} .
\end{aligned}
$$

In $z$-space the state on the right-hand side is the normalized state with $p=1$

$$
\begin{aligned}
{\left.\left[z^{1} \times \mid\left(\omega_{1} t\right) \alpha J M\right)\right]_{m_{l^{\prime \prime}}}^{t^{\prime \prime}} } & \left.\equiv\left[Z_{1}^{(10)}(z) \times \mid\left(\omega_{1} t\right) \alpha J M\right)\right]_{m_{i^{\prime}}}^{t^{\prime \prime}} \\
& \left.=\mid p=1 \omega_{1}[t \times 1] t^{\prime \prime} m_{\imath}^{\prime \prime}, \alpha J M\right) .
\end{aligned}
$$

The corresponding normalized state in standard representation is

$$
\left.\left|p=1\left(\omega_{1} t\right) t^{\prime \prime} m_{t}^{\prime \prime}, \alpha J M\right\rangle=\frac{1}{\left(K\left(1\left(\omega_{1} t\right) t^{\prime \prime}\right)\right)_{11}}\left[A_{1}^{\dagger} \times \mid \omega_{1} t, \alpha J M\right)\right]_{m_{t}^{\prime \prime}}^{t^{\prime \prime}},
$$

where the needed $K$ is given explicitly by eq. (27). Finally, using $\mathbb{a}=\Gamma(a)=$ $K \gamma(a) K^{-1}$ and the fact that $K$ acting to the left on an intrinsic state is the unit operator

$$
\begin{aligned}
\left(\left(\omega_{1}-\frac{1}{2} t^{\prime}\right) \alpha^{\prime} J^{\prime}\|\infty\|\left[z^{1} \times \mid\left(\omega_{1} t\right) \alpha J\right)\right]^{t^{\prime \prime}} \\
=\left(\left(\omega_{1}-\frac{1}{2} t^{\prime}\right) \alpha^{\prime} J^{\prime}\left\|\gamma(a) K^{-1}\right\| p=1 \omega_{1}[t \times 1] t^{\prime \prime} \alpha J\right) \\
\left.=\frac{1}{\left(K^{2}\left(1\left(\omega_{1} t\right) t^{\prime \prime}\right)\right)_{11}}\left\langle\omega_{1}-\frac{1}{2} t^{\prime} m_{t}^{\prime} \alpha^{\prime} J^{\prime} M^{\prime}\right|\left[a^{1 / 2} \times\left[A_{1}^{\dagger} \times \mid\left(\omega_{1} t\right) \alpha J M\right)\right]^{t^{\prime \prime}}\right]_{m_{r^{\prime}}}^{t^{\prime}} \\
=\frac{1}{\left(K^{2}\left(1\left(\omega_{1} t\right) t^{\prime \prime}\right)\right)_{11}} \sum_{\tau} U\left(t 1 t^{\prime} \frac{1}{2} ; t^{\prime \prime} \tau\right) \\
\quad \times\left\langle\omega_{1}-\frac{1}{2} t^{\prime} m_{t}^{\prime} \alpha^{\prime} J^{\prime} M^{\prime}\right|\left[\left[a^{1 / 2}, A_{1}^{\dagger}\right]^{\tau} \times\left|\left(\omega_{1} t\right) \alpha J M\right\rangle\right]_{m_{t^{\prime}}}^{t^{\prime}} .
\end{aligned}
$$

In the last step the vector coupled product $\left[a^{1 / 2} \times A_{1}^{\dagger}\right]_{m_{1}}^{r}$ has been converted to a vector coupled commutator, defined by

$$
\begin{aligned}
{\left[a^{1 / 2}, A_{1}^{\dagger}\right]_{m_{\tau}}^{\tau} } & =\sum_{M_{1} m_{t}}\left\langle 1 M_{T}^{\frac{1}{2}} m_{t} \mid \tau m_{\tau}\right\rangle(-1)^{j-m+\frac{1}{2}-m_{t}}\left[a_{j-m_{2}^{1}-m_{t}}, A_{1 M_{T}}^{*}\right] \\
& =\sqrt{\frac{3}{2}} a_{j m_{2} \frac{1}{\psi} m_{t}} \delta_{\tau_{2}^{1}}
\end{aligned}
$$


where straightforward anticommutation properties of the fermion operators have been used in eq. (38); and in eq. (37) we have used the fact that $A^{\dagger}$ acting to the left will annihilate the intrinsic state with $n^{\prime}=v^{\prime}$. Collecting all the results, a reduced matrix element of $a^{\dagger}$ between intrinsic states has been related to a reduced matrix element of $a^{\dagger}$, the latter to be expressed in standard representation

$$
\begin{aligned}
& \left(\left(\omega_{1}-\frac{1}{2} t^{\prime}\right) \alpha^{\prime} J^{\prime}\left\|\mathbb{N}^{\dagger}\right\|\left(\omega_{1} t\right)\right) \\
& =\left\{1+\frac{3}{2} \sum_{t^{\prime \prime}} \frac{U^{2}\left(t 1 t^{\prime} \frac{1}{2} ; t^{\prime \prime} \frac{1}{2}\right)}{\left(K^{2}\left(1\left(\omega_{1} t\right) t^{\prime \prime}\right)\right)_{11}}\right\}\left\langle\left(\omega_{1}-\frac{1}{2} t^{\prime}\right) \alpha^{\prime} J^{\prime}\left\|a^{+}\right\|\left(\omega_{1} t\right) \alpha J\right\rangle .
\end{aligned}
$$

The explicit evaluation of the $t^{\prime \prime}$ sums gives the simple result

$$
\begin{aligned}
& \left(\left(\omega_{1}-\frac{1}{2} t^{\prime}\right) \alpha^{\prime} J^{\prime}\left\|\omega^{\dagger}\right\|\left(\omega_{1} t\right) \alpha J\right) \\
& \quad=\frac{\left(2 \omega_{1}+3\right)\left(\omega_{1}+t+2\right)\left(\omega_{1}+1-t\right)}{2\left(\omega_{1}+1\right)\left(\omega_{1}-\frac{1}{2}+t^{\prime}+2\right)\left(\omega_{1}-\frac{1}{2}+1-t^{\prime}\right)}\left\langle\left(\omega_{1}-\frac{1}{2} t^{\prime}\right) \alpha^{\prime} J^{\prime}\left\|a^{\dagger}\right\|\left(\omega_{1} t\right) \alpha J\right\rangle .
\end{aligned}
$$

In eq. (40) the reduced matrix element of $a^{\dagger}$ between intrinsic states can be cxpressed in terms of a simple (known) $\mathrm{SO}(5) \supset \mathrm{U}(2)$ reduced Wigner coefficient, connecting lowest weight $\mathrm{SO}(5)$ states, and an $\mathrm{SO}(5)$ reduced matrix element

$$
\begin{aligned}
\left\langle\left(\omega_{1}-\frac{1}{2} t^{\prime}\right) \alpha^{\prime} J^{\prime}\left\|a^{\dagger}\right\|\left(\omega_{1} t\right) \alpha J\right\rangle= & \left\langle\left(\omega_{1} t\right) H_{1}=-\omega_{1} t ;\left(\frac{1}{2} \frac{1}{2}\right) \frac{1}{2} \frac{1}{2} \|\left(\omega_{1}-\frac{1}{2} t^{\prime}\right) H_{1}^{\prime}=-\left(\omega_{1}-\frac{1}{2}\right) t^{\prime}\right\rangle \\
& \times\left\langle\left(\omega_{1}-\frac{1}{2} t^{\prime}\right) \alpha^{\prime} J^{\prime}\left\|T^{(12} \frac{1}{22}\right\|\left(\omega_{1} t\right) \alpha J\right\rangle .
\end{aligned}
$$

The $S O(5)$ reduced matrix element of the $\left(\frac{1}{2} \frac{1}{2}\right)$ irreducible tensor, denoted by both double lines and double carets following the notation introduced in ref. ${ }^{7}$ ), carries all dependence on quantum numbers outside $\mathrm{SO}(5)$. Its full evaluation would thus require detailed knowledge of the $\operatorname{Sp}(2 j+1)$ structure of the $n=v$ states. However, it is not needed in the present investigation since it will always factor out of the calculation. The reduced matrix element of $a^{\dagger}$ between states of arbitrary $n, T$ (or $\left.p=\frac{1}{2}(n-v)=\omega_{1}+H_{1}, T\right)$ will also depend on this matrix element

$$
\begin{aligned}
& \left\langle p^{\prime}\left(\omega_{1}^{\prime} t^{\prime}\right) T^{\prime} i^{\prime} ; \alpha^{\prime} J^{\prime}\left\|a^{\dagger}\right\| p\left(\omega_{1} t\right) T i ; \alpha J\right\rangle \\
& \quad=\left\langle\left(\omega_{1} t\right) H_{1} T i ;\left(\frac{11}{22}\right)+\frac{1}{2} \frac{1}{2} \|\left(\omega_{1}^{\prime} t^{\prime}\right) H_{1}^{\prime} T^{\prime} i\right\rangle \times\left\langle\left(\omega_{1}^{\prime} t^{\prime}\right) \alpha^{\prime} J^{\prime}\left\|T_{22}^{(112)}\right\|\left(\omega_{1} t\right) \alpha J\right\rangle .
\end{aligned}
$$

Thus, by relating the matrix element of $a^{\dagger}$ between states of arbitrary $n, T$ to the matrix element of $\mathbb{0}^{\dagger}$ between intrinsic states as given by eqs. (40) and (41), the required $\mathrm{SO}(5) \supset \mathrm{U}(2)$ reduced Wigner coefficient can be evaluated. The $\mathrm{SO}(5) \supset$ U(2) Wigner coefficient connecting lowest weight (intrinsic) states, needed for the full evaluation of eq. (41), follows from a simple symmetry property for such coefficients (the $1 \leftrightarrow 3$ interchange symmetry in the $1 \times 2 \rightarrow 3$ coupling; for a fuller discussion of such symmetry properties, see appendix B)

$$
\begin{aligned}
\left\langle\left(\omega_{1} t\right)\right. & \left.-\omega_{1} t ;\left(\frac{11}{2}\right)+\frac{1}{2} \frac{1}{2} \|\left(\omega_{1}-\frac{1}{2} t^{\prime}\right)-\left(\omega_{1}-\frac{1}{2}\right) t^{\prime}\right\rangle \\
= & \sqrt{\frac{\operatorname{dim}\left(\omega_{1}-\frac{1}{2} t^{\prime}\right)(2 t+1)}{\operatorname{dim}\left(\omega_{1} t\right)\left(2 t^{\prime}+1\right)}}\left\langle\left(\omega_{1}-\frac{1}{2} t^{\prime}\right)-\left(\omega_{1}-\frac{1}{2}\right) t^{\prime} ;\left(\frac{11}{2}\right)-\frac{1}{2} \frac{1}{\|} \|\left(\omega_{1} t\right)-\omega_{1} t\right\rangle,
\end{aligned}
$$


where

$$
\left\langle\left(\omega_{1}-\frac{1}{2} t^{\prime}\right)-\left(\omega_{1}-\frac{1}{2}\right) t^{\prime} ;\left(\frac{1}{2} \frac{1}{2}\right)-\frac{1}{2} \frac{1}{2} \|\left(\omega_{1} t\right)-\omega_{1} t\right\rangle=+1
$$

(Except for the phase, this last coefficient is determined by the $1 \times 1$ character of the implied unitary transformation. For a discussion of phase conventions, see appendix B.) It is interesting to note that the numerical factor of eq. (40) is the inverse, without the square root, of the $S O(5)$ and $S U(2)$ dimension ratios of eq. (43). For the more challenging cases needed in this investigation, however, the numerical factors in the intrinsic operator reduced matrix elements do not have such a simple structure. A complete tabulation of the intrinsic operator reduced matrix elements used in this investigation is given in table 3 . These have been derived by the methods illustrated in detail through eqs. (34)-(40).

With the intrinsic operator reduced matrix elements given in table 3 , the $\mathrm{SO}(5) \supset$ $U(2)$ reduced Wigner coefficients can now be calculated. The method of calculation will be illustrated in detail for the general coupling involving the 4-dimensional representation, $\left(\omega_{1} t\right) \times\left(\frac{1}{2} \frac{1}{2}\right) \rightarrow\left(\omega_{1}^{\prime} t^{\prime}\right)$. All that remains to be done is to relate the matrix elements of $a^{+}$, (or $a$ ), between states of arbitrary $n, T$ to the matrix elements of $\mathbb{a}^{\dagger},($ or $\mathbb{0}$ ), between intrinsic states. This involves essentially nothing more than some angular-momentum recoupling. The simplest calculation involves the case $\omega_{1}^{\prime}=\omega_{1}+\frac{1}{2}$ where the operator $a^{\dagger}$ (or $a$ ), reduces the seniority number, i.e. $v^{\prime}=v-1$. E.g.

$$
\begin{aligned}
& \left\langle p^{\prime}=p+1\left(\omega_{1}+\frac{1}{2} t^{\prime}\right) T^{\prime} i^{\prime} ; \alpha^{\prime} J^{\prime}\left\|a^{\dagger}\right\| p\left(\omega_{1} t\right) T i ; \alpha J\right\rangle \\
& \left.\left.=\left\langle\left(\omega_{1} t\right) H_{1} T i ;\left(\frac{1}{2} \frac{1}{2}\right)+\frac{1}{2} \frac{1}{2} \|\left(\omega_{1}+\frac{1}{2} t^{\prime}\right) H_{1}^{\prime} T^{\prime} i^{\prime}\right\rangle\left\langle\left(\omega_{1}+\frac{1}{2} t^{\prime}\right) \alpha^{\prime} J^{\prime} \| T^{(12} 2\right) \|\left(\omega_{1} t\right) \alpha J\right\rangle\right\rangle \\
& =\left(p+1\left(\omega_{1}+\frac{1}{2} t^{\prime}\right) T^{\prime} i^{\prime} ; \alpha^{\prime} J^{\prime}\left\|\gamma\left(a^{\dagger}\right)\right\| p\left(\omega_{1} t\right) T i ; \alpha J\right) \\
& =\sum_{T_{p}^{\prime}, T_{p}}\left(K^{-1}\left(p+1\left(\omega_{1}+\frac{1}{2} t^{\prime}\right) T^{\prime}\right)\right)_{t_{T_{p}^{\prime}}}\left(K\left(p\left(\omega_{1} t\right) T\right)\right)_{l_{p^{\prime}}} \\
& \times\left\{\left(p+1 \omega_{1}+\frac{1}{2}\left[t^{\prime} \times T_{p}^{\prime}\right] T^{\prime} ; \alpha^{\prime} J^{\prime}\left\|\Phi^{\dagger}\right\| p \omega_{1}\left[t \times T_{p}\right] T ; \alpha J\right)\right. \\
& \left.+\sqrt{\frac{3}{2}}\left(p+1 \omega_{1}+\frac{1}{2}\left[t^{\prime}+T_{p}^{\prime}\right] T^{\prime} ; \alpha^{\prime} J^{\prime}\left\|\left[z^{1} \times \mathbb{w}^{1 / 2}\right]^{1 / 2}\right\| p \omega_{1}\left[t \times T_{p}\right] T ; \alpha J\right)\right\},
\end{aligned}
$$

where eqs. (30c) and (30b) have been used. Since the left action of the intrinsic operator $a^{\dagger}$ on a state with $v^{\prime}=v-1$ would lower the seniority even further the first term in \{\} vanishes; (see also entry 1 of table 3). Straightforward recoupling transforms the second term into

$$
\begin{aligned}
\sqrt{\frac{3}{2}}(p+ & \left.1 \omega_{1}+\frac{1}{2}\left[t^{\prime} \times T_{p}^{\prime}\right] T^{\prime} ; \alpha^{\prime} J^{\prime}\left\|\left[z^{1} \times \mathbb{w}^{1 / 2}\right]^{1 / 2}\right\| p \omega_{1}\left[t \times T_{p}\right] T ; \alpha J\right) \\
= & \sqrt{\frac{3}{2}}\left[\begin{array}{ccc}
t & T_{p} & T \\
\frac{1}{2} & 1 & \frac{1}{2} \\
t^{\prime} & T_{p}^{\prime} & T^{\prime}
\end{array}\right] \sqrt{(p+1)}\left\langle(p 0) T_{p} ;(10) 1 \|(p+1,0) T_{p}^{\prime}\right\rangle \\
& \times\left\langle\left(\omega_{1}+\frac{1}{2} t^{\prime}\right) \alpha^{\prime} J^{\prime}\|a\|\left(\omega_{1} t\right) \alpha J\right\rangle,
\end{aligned}
$$


TABLE 3

Catalogue of intrinsic-operator reduced matrix elements

1. $\left(\left(\omega_{1}+\frac{1}{2} t^{\prime}\right) \alpha^{\prime} J^{\prime}\left\|\mathbf{a}^{\dagger}\right\|\left(\omega_{1} t\right) \alpha J\right)=0$

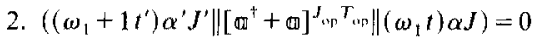

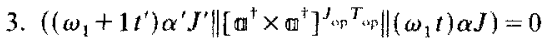

4. $\left(\left(\omega_{1}-\frac{1}{2} t^{\prime}\right) \alpha^{\prime} J^{\prime}\left\|\mathbb{\Phi}^{\dagger}\right\|\left(\omega_{1} t\right) \alpha J\right)=\frac{\left(2 \omega_{1}+3\right)\left(\omega_{1}+t+2\right)\left(\omega_{1}+1-t\right)}{2\left(\omega_{1}+1\right)\left(\omega_{1}+t^{\prime}+\frac{3}{2}\right)\left(\omega_{1}+\frac{1}{2}-t^{\prime}\right)}\left\langle\left(\omega_{1}-\frac{1}{2} t^{\prime}\right) \alpha^{\prime} J^{\prime}\left\|a^{\dagger}\right\|\left(\omega_{1} t\right) \alpha J\right\rangle$

5. $\left(\left(\omega_{1}+\frac{1}{2} t^{\prime}\right) \alpha^{\prime} J^{\prime}\left\|\sigma \sigma_{1}\right\|\left(\omega_{1} t\right) \alpha J\right)=\left\langle\left(\omega_{1}+\frac{1}{2} t^{\prime}\right) \alpha^{\prime} J^{\prime}\|a\|\left(\omega_{1} t\right) \alpha, J\right\rangle$

6. $\left(\left(\omega_{1}-\frac{1}{2} t^{\prime}\right) \alpha^{\prime} J^{\prime}\|\bowtie\| p=1 \omega_{1}[t \times 1] t^{\prime \prime} \alpha J\right)$

$$
=\sqrt{\frac{3}{2}} \frac{U\left(t 1 t^{\prime} \frac{1}{2} ; t^{\prime \prime} \frac{1}{2}\right)}{\left(K^{2}\left(1\left(\omega_{1} t\right) t^{\prime \prime}\right)\right)_{11}}\left\langle\left(\omega_{1}-\frac{1}{2} t^{\prime}\right) \alpha^{\prime} J^{\prime}\left\|a^{\dagger}\right\|\left(\omega_{1} t\right) \alpha J\right\rangle
$$

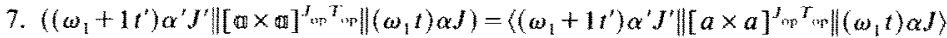

8. $\left(\left(\omega_{1} t^{\prime}\right) \alpha^{\prime} J^{\prime}\left\|\sqrt{\frac{1}{2}}[\square \times \infty]^{3}{ }^{0}\right\| p=1 \omega_{1}[t \times 1] t^{\prime} \alpha J\right)$

$$
=-\frac{1}{\left(K^{2}\left(1\left(\omega_{1} t\right) t^{\prime}\right)\right)_{11}}\left\langle\left(\omega_{1} t^{\prime}\right) \alpha^{\prime} J^{\prime}\left\|\left[a^{+} \times a\right]^{t^{\prime}{ }^{1}}\right\|\left(\omega_{1} t\right) \alpha J\right\rangle
$$

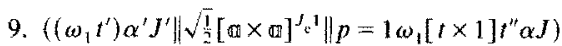

$$
\begin{aligned}
= & \frac{1}{\left(K^{2}\left(1\left(\omega_{1} t\right) t^{\prime \prime}\right)\right)_{11}}\left\{(-1)^{t+1-t^{\prime \prime}} \sqrt{\frac{2 t^{\prime \prime}+1}{2 t+1}}\right. \\
& \times\left\langle\left(\omega_{1} t^{\prime}\right) \alpha^{\prime} J^{\prime}\left\|\left[a^{+} \times a\right]^{\prime \prime \prime}\right\|\left(\omega_{1} t\right) \alpha J\right\rangle \delta_{t^{\prime},} \\
& \left.+\sqrt{2} U\left(t 1 t^{\prime} 1 ; t^{\prime \prime} 1\right)\left\langle\left(\omega_{1} t^{\prime}\right) \alpha^{\prime} J^{\prime}\left\|\left[a^{\dagger} \times a\right]^{J^{1}}\right\|\left(\omega_{1} t\right) \alpha J\right\rangle\right\}
\end{aligned}
$$

10. $\left(\left(\omega_{1} t^{\prime}\right) \alpha^{\prime} J^{\prime}\left\|\left[a^{+} \times a\right]^{s_{3}}\right\|\left(\omega_{1} t\right) \alpha J\right)=\frac{\left[1+\left(K^{2}\left(1\left(\omega_{1} t\right) t^{\prime}\right)\right)_{11}\right]}{\left(K^{2}\left(1\left(\omega_{1} t\right) t^{\prime}\right)\right)_{11}}\left\langle\left(\omega_{1} t^{\prime}\right) \alpha^{\prime} J\left\|\left[a^{+} \times a\right]^{J^{1}}\right\|\left(\omega_{1} t\right) \alpha J\right\rangle$

11. $\left(\left(\omega_{1} f^{\prime}\right) \alpha^{\prime} J^{\prime}\left\|\left[\sigma^{+} \times \infty\right]^{e^{\prime}}\right\|\left(\omega_{1} f\right) \alpha J\right)$

$$
\begin{aligned}
= & \left\{1+2 \sum_{t^{\prime \prime}} \frac{U^{2}\left(t 1 t^{\prime} 1 ; t^{\prime \prime} 1\right)}{\left(K^{2}\left(1\left(\omega_{1} t\right) t^{\prime \prime}\right)\right)_{11}}\right\}\left\langle\left(\omega_{1} t^{\prime}\right) \alpha^{\prime} J^{\prime} \|\left[a^{+} \times a\right]^{J_{\mathrm{e}}{ }^{1}} \mid\left(\omega_{1} t\right) \alpha J\right\rangle \\
& \left.+\sqrt{2} \delta_{t^{\prime},} \sum_{t^{\prime}} \frac{(-1)^{t+1-t^{\prime \prime}} \sqrt{\left(2 t^{\prime \prime}+1\right)} U\left(t 1 t 1 ; t^{\prime \prime} 1\right)}{\left(K^{2}\left(1\left(\omega_{1} t\right) t^{\prime \prime}\right)\right)_{1} \sqrt{(2 t+1)}}\left\langle\left(\omega_{1} t\right) \alpha^{\prime} J^{\prime}\left\|\left[a^{+} \times a\right]^{J_{\mathrm{e}} 0}\right\|\left(\omega_{1} t\right) \alpha J\right\rangle\right\}
\end{aligned}
$$

12. $\left((\omega, t) \alpha^{\prime} J^{\prime}\left\|\left[\alpha^{*} \times \infty\right]^{3, y}\right\|(\omega, t) \alpha J\right)$

$$
\begin{aligned}
& =\left\{1+\sum_{t^{\prime \prime}} \frac{\left(2 t^{\prime \prime}+1\right)}{(2 t+1)\left(K^{2}\left(1\left(\omega_{1} t\right) t^{\prime \prime}\right)\right)_{11}}\right\}\left\langle\left(\omega_{1} t\right) \alpha^{\prime} J^{\prime}\left\|\left[a^{\dagger} \times a\right]^{J_{\mathrm{c}}{ }^{\prime}}\right\|\left(\omega_{1} t\right) \alpha J\right\rangle \\
& \left.\quad+\sqrt{2} \sum_{i^{\prime \prime}} \frac{(-1)^{\prime+1-t^{\prime \prime}} \sqrt{\left(2 t^{\prime \prime}+1\right)} U\left(t 1 t 1 ; t^{\prime \prime} 1\right)}{\left(K^{2}\left(1\left(\omega_{1} t\right) t^{\prime \prime}\right)\right)_{11} \sqrt{(2 t+1)}}\left\langle\left(\omega_{1} t\right) \alpha^{\prime} J^{\prime} \|\left[a^{+} \times a\right]^{J}\right]^{\prime} \|\left(\omega_{1} t\right) \alpha J\right\rangle
\end{aligned}
$$

where

$$
\begin{aligned}
& 1+\sum_{t^{\prime \prime}} \frac{\left(2 t^{\prime \prime}+1\right)}{(2 t+1)\left(K^{2}\left(1\left(\omega_{1}+1\right)^{2} t^{\prime \prime}\right)\right)_{11}}=\frac{\left(\omega_{1}+1\right)^{2}\left(\omega_{1}+3\right)-\left(\omega_{1}+2\right) t(t+1)}{\left(\omega_{1}-t\right)\left(\omega_{1}+1\right)\left(\omega_{1}+t+1\right)} \\
& \sqrt{ } 2 \sum_{t^{\prime \prime}} \frac{(-1)^{t+1-r^{\prime \prime}} \sqrt{\left(2 t^{\prime \prime}+1\right)} U\left(t 1 t 1: t^{\prime \prime} 1\right)}{\left(K^{2}\left(1\left(\omega_{1} t\right) t^{\prime \prime}\right)\right)_{11} \sqrt{(2 t+1)}}=\frac{\left(2 \omega_{1}+3\right) \sqrt{t(t+1)}}{\left(\omega_{1}-t\right)\left(\omega_{1}+1\right)\left(\omega_{1}+t+1\right)} \\
& 1+2 \sum_{t^{\prime \prime}} \frac{U^{2}\left(t 1 t^{\prime} 1 ; t^{\prime \prime} 1\right)}{\left.\left(1\left(\omega_{1} t\right) t^{\prime \prime}\right)\right)_{11}}= \begin{cases}\frac{\left(\omega_{1}+2\right)\left(\omega_{1}+1-t\right)}{\left(\omega_{1}+1\right)\left(\omega_{1}-t\right)} & \text { for } t^{\prime}=t+1 \\
\frac{\left(\omega_{1}+2\right)\left(\omega_{1}+2+t\right)}{\left(\omega_{1}+1\right)\left(\omega_{1}+1+t\right)} & \text { for } t^{\prime}=t-1 \\
\frac{\omega_{1}\left(\omega_{1}+2\right)^{2}-\left(\omega_{1}+1\right) t(t+1)}{\left(\omega_{1}-t\right)\left(\omega_{1}+1\right)\left(\omega_{1}+t+1\right)} & \text { for } t^{\prime}=t\end{cases}
\end{aligned}
$$


where eq. (19) and entry 5 of table 3 have been used for the reduced matrix elements of $z$ and $₫$. Note that eq. (19) could also be expressed through the useful identity

$$
\left[z^{1} \times Z_{T_{p}}^{(p 0)}(z)\right]_{T_{p}^{\prime} M i_{r}}=Z_{T_{p}, M T_{p}}^{(p+1,0)}(z) \sqrt{(p+1)}\left\langle(p 0) T_{p} ;(10) 1 \|(p+1,0) T_{p}^{\prime}\right\rangle .
$$

Finally,

$$
\begin{aligned}
& \left\langle\left(\omega_{1}+\frac{1}{2} t^{\prime}\right) \alpha^{\prime} J^{\prime}\|a\|\left(\omega_{1} t\right) \alpha J\right\rangle \\
& \quad=\left\langle\left(\omega_{1} t\right)-\omega_{1} t ;\left(\frac{11}{22}\right)-\frac{1}{22} \|\left(\omega_{1}+\frac{1}{2} t^{\prime}\right)-\left(\omega_{1}+\frac{1}{2}\right) t^{\prime}\right\rangle\left\langle\left(\omega_{1}+\frac{1}{2} t^{\prime}\right) \alpha^{\prime} J^{\prime}\left\|T_{22}^{\left(\frac{1}{2}\right)}\right\|\left(\omega_{1} t\right) \alpha J\right\rangle,
\end{aligned}
$$

where the $\mathrm{SO}(5)$ Wigner coefficient connecting intrinsic (or $\mathrm{SO}(5)$ lowest weight) states has the simple value +1 . The combination of eqs. (45)-(46) gives the wanted $\mathrm{SO}(5) \supset \mathrm{U}(2)$ Wigner coefficient which is writen explicitly as the first energy of table 4. Note that this Wigner coefficient is given solely by the $K$-matrix elements of vector coherent state theory, a very simple 3-dimensional oscillator coupling coefficient, and an ordinary angular momentum $9-j$ recoupling coefficient, given here in unitary (square bracket) form. The second entry in table 4 follows in similar

TABLE 4

$\mathrm{SO}(5) \supset \mathrm{U}(2)$ Wigner coefficients for the coupling $\left(\omega_{1} t\right) \times\left(\frac{1}{2} \frac{1}{2}\right) \rightarrow\left(\omega_{1}^{\prime} t^{\prime}\right)$

Case 1. $\left(\omega_{1}^{\prime} t^{\prime}\right)=\left(\omega_{1}+\frac{1}{2}, t^{\prime}\right) ; v^{\prime}=v-1$

$\left\langle\left(\omega_{1} t\right) H_{1} T_{i} ;\left(\begin{array}{l}1 \\ 2 \\ 2\end{array}\right) h_{1} \frac{1}{2} \|\left(\omega_{1}^{\prime} t^{\prime}\right) H_{1}^{\prime}=H_{1}+h_{1} T^{\prime} i^{\prime}\right\rangle$

$=\sum_{I_{p^{\prime},}, T_{p}^{\prime}}\left(K^{\prime}\left(p^{\prime}\left(\omega_{1}^{\prime} t^{\prime}\right) T^{\prime}\right)\right)_{i} T_{p}^{\prime}\left(K\left(p\left(\omega_{1} t\right) T\right)\right)_{T_{p i}} F\left(h_{1} ; T T_{p} ; T^{\prime} T_{p}^{\prime}\right)$

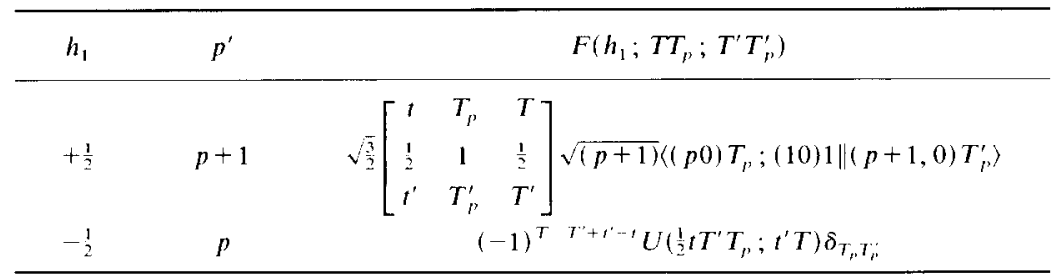

Case 2. $\left(\omega_{1}^{\prime} t^{\prime}\right)=\left(\omega_{1}-\frac{1}{2}, t^{\prime}\right) ; v^{\prime}=v+1$

$\left\langle\left(\omega_{1} t\right) H_{1} T i ;\left(\frac{1}{2} \frac{1}{2}\right) h_{1} \frac{1}{2} \|\left(\omega_{1}^{\prime} t^{\prime}\right) H_{1}+h_{1} T^{\prime} i^{\prime}\right\rangle$

$=\sum_{I_{p}, t_{n}}\left(K^{-1}\left(p\left(\omega_{1} t\right) T\right)\right)_{i T_{p}}\left(K\left(p^{\prime}\left(\omega_{1}^{\prime} t^{\prime}\right) T^{\prime}\right)\right)_{T_{p},} F\left(h_{1} ; T T_{p} ; T^{\prime} T_{p}^{\prime}\right)$

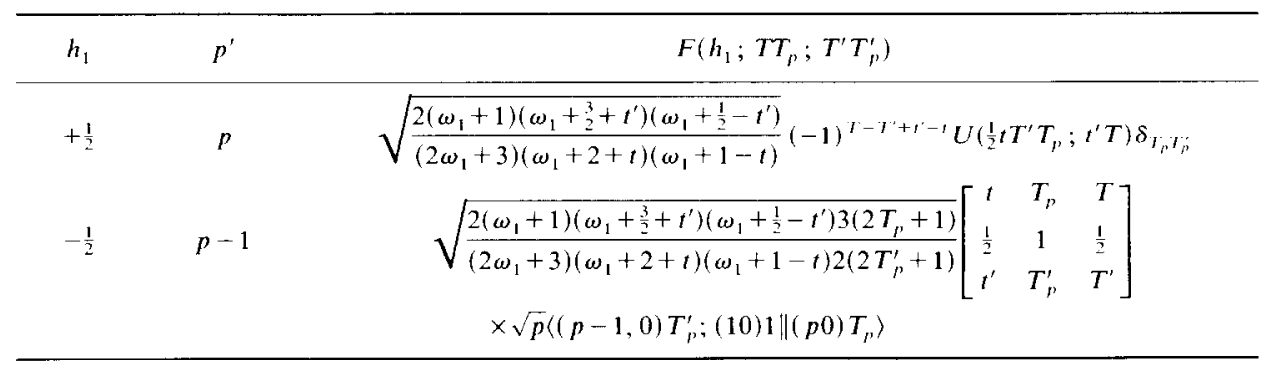


fashion from the reduced matrix element of $a$. Since, $\Gamma(a)=\mathbb{a}$, contains but a single term, and $p^{\prime}=p$ in this case, the recoupling process is even simpler and leads to an expression involving an ordinary Racah coefficient given here in U-coefficient that is again in unitary form.

Coefficients for $\omega_{1}^{\prime}=\omega_{1}-\frac{1}{2}$ for which $a^{i}$ (or $a$ ) increases the seniority number from $v$ to $v^{\prime}=v+1$, can be obtained in their simplest form from the coefficients with $\omega_{1}^{\prime}=\omega_{1}+\frac{1}{2}$ and the $1 \leftrightarrow 3$ interchange symmetry property for such coefficients

$$
\begin{aligned}
& \left\langle\left(\omega_{1} t\right) H_{1} T i ;\left(\frac{1}{2} \frac{1}{2}\right) h_{2}^{\frac{1}{2}} \|\left(\omega_{1}-\frac{1}{2} t^{\prime}\right) H_{1}^{\prime} T^{\prime} i^{\prime}\right\rangle \\
& =\sqrt{\frac{\operatorname{dim}\left(\omega_{1}-\frac{1}{2}, t^{\prime}\right)(2 T+1)}{\operatorname{dim}\left(\omega_{1} t\right)\left(2 T^{\prime}+1\right)}}(-1)^{T-T^{\prime}+t^{\prime}-t}\left\langle\left(\omega_{1}-\frac{1}{2} t^{\prime}\right) H_{1}^{\prime} T^{\prime} i^{\prime} ;\left(\frac{1}{2} \frac{1}{2}\right)\right. \\
& \\
& \left.\quad-h \frac{1}{2} \|\left(\omega_{1} t\right) H_{1} T i\right\rangle
\end{aligned}
$$

(see also appendix B). With the renaming $T \leftrightarrow T^{\prime}, t \leftrightarrow t^{\prime}$, and the use of simple symmetry properties of the recoupling coefficients these can be put into the simple form given in table 4 .

Although coefficients for $\omega_{1}^{\prime}=\omega_{1}-\frac{1}{2}$ can be obtained in their simplest form from this symmetry property they can also be evaluated directly by the techniques outlined above. Thus, e.g.

$$
\begin{aligned}
\left\langle p^{\prime}=\right. & \left.p-1\left(\omega_{1}-\frac{1}{2} t^{\prime}\right) T^{\prime} i^{\prime} ; \alpha^{\prime} J^{\prime}\|a\| p\left(\omega_{1} t\right) T i ; \alpha J\right\rangle \\
= & \sum_{T_{p^{\prime},}^{\prime}, T_{p}}\left(K^{-1}\left(p-1\left(\omega_{1}-\frac{1}{2} t^{\prime}\right) T^{\prime}\right)\right)_{i^{\prime} T_{p}^{\prime}}\left(K\left(p\left(\omega_{1} t\right) T\right)\right)_{T_{p}, i} \\
& \quad \times\left(p-1 \omega_{1}-\frac{1}{2}\left[t \times T_{p}^{\prime}\right] T^{\prime} ; \alpha^{\prime} J^{\prime}\|\mathbb{ه}\| p \omega_{1}\left[t \times T_{p}\right] T ; \alpha J\right)
\end{aligned}
$$

again with the use of eqs. (30c) and (30a). Although care must be taken to define the reduced matrix element of $\infty$ through its left action (see entry 6 of table 3 ) we will find it more convenient to perform the angular momentum recoupling on the right through prior hermitian conjugation, and use

$$
\begin{aligned}
& \left(p-1 \omega_{1}-\frac{1}{2}\left[t^{\prime} \times T_{p}^{\prime}\right] T^{\prime} ; \alpha^{\prime} J^{\prime}\left\|\varpi_{j}^{1}\right\| p \omega_{1}\left[t \times T_{p}\right] T ; \alpha J\right) \\
& =(-1)^{J+j-J^{\prime}+T+\frac{1}{2}-T^{\prime}} \sqrt{\frac{(2 J+1)(2 T+1)}{\left(2 J^{\prime}+1\right)\left(2 T^{\prime}+1\right)}} \\
& \times\left(p \omega_{1}\left[t \times T_{p}\right] T M_{T} ; \alpha J M \mid\left[{\varpi_{j 2}^{\prime}}_{j}^{\dagger} \times\left[Z_{T_{p}^{\prime}}^{(p-1.0)}(z) \times \mid\left(\omega_{1}-\frac{1}{2} t^{\prime}\right) ; \alpha^{\prime} J^{\prime}\right)\right]^{T^{\prime}}\right]_{M M_{I}}^{J T^{*}} \\
& =(-1)^{J+j-J^{\prime}+T+\frac{1}{2}-T^{\prime}} \sqrt{\frac{(2 J+1)(2 T+1)}{\left(2 J^{\prime}+1\right)\left(2 T^{\prime}+1\right)}} \sum_{t^{\prime \prime}}(-1)^{T-T^{\prime}+t^{\prime}-t^{\prime \prime}} U\left(\frac{1}{2} t^{\prime} T T_{p}^{\prime} ; t^{\prime \prime} T^{\prime}\right) \\
& \times\left(p \omega_{1}\left[t \times T_{p}\right] T M_{T} \mid\left[Z_{T_{p}^{\prime}}^{p-1,0}(z) \times\left[z^{1} \times \mid \omega_{1} t\right)\right]^{t^{\prime \prime}}\right]_{M T}^{T^{*}} \\
& \times\left(p=1 \omega_{1}[t \times 1] t^{\prime \prime} ; \alpha J\left\|\mathbb{Q}_{j_{2}}^{+}\right\|\left(\omega_{1}-\frac{1}{2} t^{\prime}\right) ; \alpha^{\prime} J^{\prime}\right)^{*},
\end{aligned}
$$


where the intrinsic-operator matrix element will be reinverted by hermitian conjugation

$$
\begin{aligned}
(p=1 & \left.1 \omega_{1}[t \times 1] t^{\prime \prime} ; \alpha J\left\|\Phi_{j_{2}^{\prime}}^{\prime}\right\|\left(\omega_{1}-\frac{1}{2} t^{\prime}\right) \alpha^{\prime} J^{\prime}\right)^{*} \\
= & (-1)^{J+j-J^{\prime}+t^{\prime \prime}+\frac{1}{2}-t^{\prime}} \sqrt{\frac{\left(2 J^{\prime}+1\right)\left(2 t^{\prime}+1\right)}{(2 J+1)\left(2 t^{\prime \prime}+1\right)}} \\
& \times\left(\left(\omega_{1}-\frac{1}{2} t^{\prime}\right) \alpha^{\prime} J^{\prime}\left\|\Phi_{j_{2}}\right\| p=1 \omega_{1}[t \times 1] t^{\prime \prime} ; \alpha J\right),
\end{aligned}
$$

so that the right-hand side can be read from entry 6 of table 3 . The $z$-space overlap is given by

$$
\begin{aligned}
& \left(p \omega_{1}\left[t \times T_{p}\right] T M_{T} \mid\left[Z_{T_{p}^{\prime}}^{(p-1,0)}(z) \times\left[z^{1} \times \mid \omega_{1} t\right)\right]^{\prime \prime}\right]_{M_{T}}^{T} \\
& \quad=U\left(t 1 T T_{p}^{\prime} ; t^{\prime \prime} T_{p}\right) \sqrt{p}\left\langle(p-1,0) T_{p}^{\prime} ;(10) 1 \|(p 0) T_{p}\right\rangle .
\end{aligned}
$$

Finally, combining all terms, using the analogue of eq. (41) and the simple matrix element

$$
\begin{aligned}
\left\langle\left(\omega_{1}-\frac{1}{2} t^{\prime}\right) \alpha^{\prime} J^{\prime}\left\|a^{\dagger}\right\|\left(\omega_{1} t\right) \alpha J\right\rangle \\
\left.\quad=\left\langle\left(\omega_{1} t\right)-\omega_{1} t ;\left(\frac{11}{2}\right)+\frac{1}{2} \frac{1}{2} \|\left(\omega_{1}-\frac{1}{2} t^{\prime}\right)-\left(\omega_{1}-\frac{1}{2}\right) t^{\prime}\right\rangle\left\langle\left(\omega_{1}-\frac{1}{2} t^{\prime}\right) \alpha^{\prime} J^{\prime}\left\|T^{\left(\frac{1}{22}\right)}\right\|\left(\omega_{1} t\right) \alpha J\right\rangle\right\rangle \\
=\sqrt{\frac{2\left(\omega_{1}+1\right)\left(\omega_{1}+\frac{3}{2}+t^{\prime}\right)\left(\omega_{1}+\frac{1}{2}-t^{\prime}\right)}{\left(2 \omega_{1}+3\right)\left(\omega_{1}+2+t\right)\left(\omega_{1}+1-t\right)}}\left\langle\left(\omega_{1}-\frac{1}{2} t^{\prime}\right) \alpha^{\prime} J^{\prime}\left\|T^{(1 ! 2)}\right\|\left(\omega_{1} t\right) \alpha J\right\rangle,
\end{aligned}
$$

the $\mathrm{SO}(5)$ Wigner coefficient can be put in the form

$$
\begin{aligned}
\left\langle\left(\omega_{1} t\right)\right. & \left.H_{1} T i ;\left(\frac{11}{2}\right)-\frac{1}{2} \frac{1}{2} \|\left(\omega_{1}-\frac{1}{2} t^{\prime}\right) H_{1}^{\prime} T^{\prime} i^{\prime}\right\rangle \\
= & \sum_{T_{p}, T_{p}^{\prime}}\left(K^{-1}\left(p-1\left(\omega_{1}-\frac{1}{2} t^{\prime}\right) T^{\prime}\right)\right)_{i^{\prime} T_{r^{\prime}}}\left(K\left(p\left(\omega_{1} t\right) T\right)\right)_{T_{p},} \\
& \times \sum_{t^{\prime \prime}} \sqrt{\frac{3(2 T+1)\left(2 t^{\prime}+1\right)}{2\left(2 T^{\prime}+1\right)\left(2 t^{\prime \prime}+1\right)}} U\left(\frac{1}{2} t^{\prime} T T_{p}^{\prime} ; t^{\prime \prime} T^{\prime}\right) U\left(t 1 T T_{p}^{\prime} ; t^{\prime \prime} T_{p}\right) \\
& \times U\left(t 1 t^{\prime} \frac{1}{2} ; t^{\prime \prime} \frac{1}{2}\right) \sqrt{p}\left\langle(p-1,0) T_{p}^{\prime} ;(10) 1 \|(p 0) T_{p}\right\rangle \\
& \times \frac{1}{\left(K^{2}\left(1\left(\omega_{1} t\right) t^{\prime \prime}\right)\right)_{11}} \sqrt{\frac{2\left(\omega_{1}+1\right)\left(\omega_{1}+\frac{3}{2}+t^{\prime}\right)\left(\omega_{1}+\frac{1}{2}-t^{\prime}\right)}{\left(2 \omega_{1}+3\right)\left(\omega_{1}+2+t\right)\left(\omega_{1}+1-t\right)}} .
\end{aligned}
$$

Note that this form is somewhat more cumbersome than that given by the last enetry of table 4 . Note also that the representations $\left(\omega_{1} t\right)$ and $\left(\omega_{1}-\frac{1}{2} t^{\prime}\right)$ have exchanged places in $K$ and $K^{-1}$. The equivalence of the two results leads to the following relation

$$
\begin{aligned}
\sum_{i}[( & \left.\left.K\left(p\left(\omega_{1} t\right) T\right)\right)_{T_{p}}\right]^{2} \sum_{t^{\prime \prime}} \sqrt{\frac{(2 T+1)\left(2 t^{\prime}+1\right)}{\left(2 T^{\prime}+1\right)\left(2 t^{\prime \prime}+1\right)}} \frac{1}{\left(K^{2}\left(1\left(\omega_{1} t\right) t^{\prime \prime}\right)\right)_{11}} \\
& \times U\left(\frac{1}{2} t^{\prime} T T_{p}^{\prime} ; t^{\prime \prime} T^{\prime}\right) U\left(t 1 T T_{p}^{\prime} ; t^{\prime \prime} T_{p}\right) U\left(t 1 t^{\prime} \frac{1}{2} ; t^{\prime \prime} \frac{1}{2}\right) \\
= & \sum_{i^{\prime}}\left[\left(K\left(p-1\left(\omega_{1}-\frac{1}{2} t^{\prime}\right) T^{\prime}\right)\right)_{\left.T_{p^{\prime}}\right]^{2}}\right]^{2} \sqrt{\frac{2 T_{p}+1}{2 T_{p}^{\prime}+1}}\left[\begin{array}{ccc}
t & T_{p} & T \\
\frac{1}{2} & 1 & \frac{1}{2} \\
t^{\prime} & T_{p}^{\prime} & T^{\prime}
\end{array}\right] .
\end{aligned}
$$


With special choices of quantum numbers for which both $K$ 's become 1dimensional, this could lead to new relations among angular momentum recoupling coefficients. On the other hand, this equation might be very useful as a check on $K$-matrix element calculations.

\section{Coupling with the representations (10) and (11)}

The $\mathrm{SO}(5) \supset \mathrm{U}(2)$ reduced Wigner coefficients for the coupling with 5-dimensional representation (10) follow at once from the methods outlined in sect. 3. Results are collected in table 5. The simplest expressions occur for the coupling $\left(\omega_{1} t\right) \times(10) \rightarrow$ $\left(\omega_{1}^{\prime} t^{\prime}\right)$ with $\left(\omega_{1}^{\prime} t^{\prime}\right)=\left(\omega_{1}+1 t\right)$, i.e. for the coupling to representations with $v^{\prime}=v-2$. The coefficients for the coupling to representations $\left(\omega_{1}^{\prime} t^{\prime}\right)=\left(\omega_{1}-1 t\right)$, with $v^{\prime}=v+2$, follow from these through the $1 \leftrightarrow 3$ interchange symmetry property of such Wigner coefficients (see appendix B), and are therefore not explicitly tabulated. Similarly, the coefficient for $\left(\omega_{1}^{\prime} t^{\prime}\right)=\left(\omega_{1} t^{\prime}\right)$ with $h_{1}, \tau=+1,0$ is related to that with $h_{1}, \tau=-1,0$ through this same relation. For $\left(\omega_{1}^{\prime} t^{\prime}\right)=\left(\omega_{1} t^{\prime}\right)$ the needed starting coefficient, coupling lowest weight states, is not known ab initio. However, since our general formulae relate all coefficients to this starting coefficient, the ratios of coefficients with $H_{1}^{\prime} T^{\prime}$

TABLE 5

Table of $\mathrm{SO}(5) \supset \mathrm{U}(2)$ Wigner coefficients for the coupling $\left(\omega_{1} t\right) \times(10) \rightarrow\left(\omega_{1}^{\prime} t^{\prime}\right)$

$$
\begin{aligned}
& \left\langle\left(\omega_{1} t\right) H_{1} T i ;(10) h_{1} r \|\left(\omega_{1}^{i} t^{\prime}\right) H_{1}^{\prime}=H_{1}+h_{1} T^{\prime} i^{\prime}\right\rangle \\
& =\sum_{T_{p}, T_{p}^{\prime}}\left(K^{\prime \prime 1}\left(p^{\prime}\left(\omega_{1}^{\prime} t^{\prime}\right) T^{\prime}\right)\right)_{i^{\prime} T_{i}^{\prime}}\left(K\left(p\left(\omega_{1} t\right) T\right)\right)_{T_{i}, i} F\left(h_{1} \tau ; T T_{p} ; T^{\prime} T_{p}^{\prime}\right)
\end{aligned}
$$

Case 1. $\left(\omega_{1}^{\prime} t^{\prime}\right)=\left(\omega_{1}+1 t\right) ; v^{\prime}=v-2$

\begin{tabular}{rccc}
\hline$h_{1}$ & $\tau$ & $p^{\prime}$ & $F\left(h_{1} \tau ; T T_{n} ; T^{\prime} T_{p}^{\prime}\right)$ \\
\hline-1 & 0 & $p$ & $\delta_{T_{p} T_{p}} \delta_{T T^{\prime}}$ \\
0 & 1 & $p+1$ & $U\left(t T_{p} T^{\prime} 1 ; T T_{p}^{\prime}\right) \sqrt{(p+1)}\left((p 0) T_{p} ;(10) 1 \|(p+1,0) T_{p}^{\prime}\right\rangle$ \\
+1 & 0 & $p+2$ & $\frac{1}{2} \delta_{T_{p} r_{p}^{\prime}} \delta_{T^{\prime}, \sqrt{\left(p+T_{p}+3\right)\left(p-T_{p}+2\right)}}$ \\
\hline
\end{tabular}

Case 2. $\left(\omega_{1}^{\prime} t^{\prime}\right)=\left(\omega_{1} t^{\prime}\right) ; t^{\prime}=t \pm 1, t ; v^{\prime}=v$

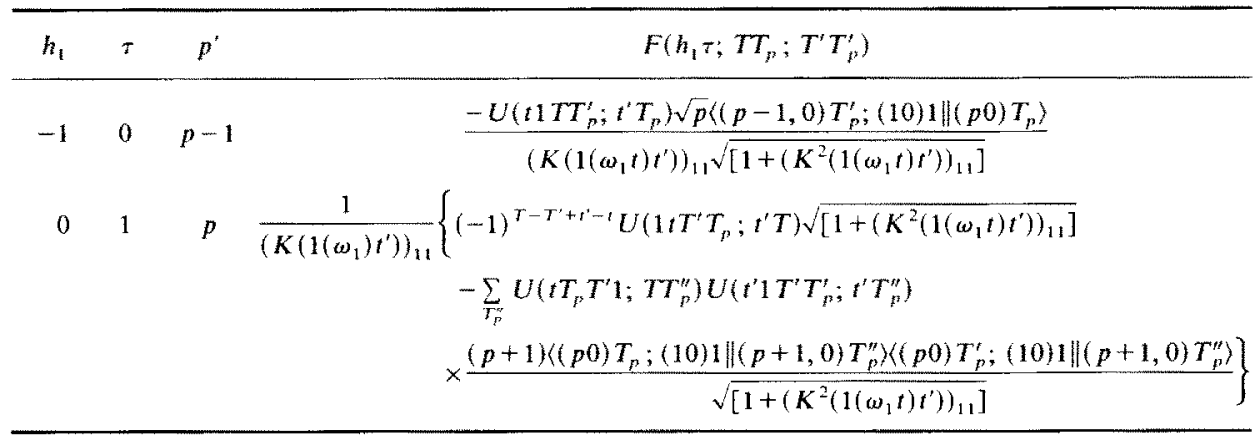


fixed at $H_{1}^{\prime}=-\omega_{1}^{\prime} T^{*}=t^{\prime}$ are given, so that the starting coefficient can be evaluated (to within a phase) from the orthonormality of the Wigner coefficients. For the case $\left(\omega_{1}^{\prime} t^{\prime}\right)=\left(\omega_{1} t^{\prime}\right)$ it has the value

$$
C_{c, \mathrm{w} .} \equiv\left\langle\left(\omega_{1} t\right)-\omega_{1} t ;(10) 01 \|\left(\omega_{1} t^{\prime}\right)-\omega_{1} t^{\prime}\right\rangle=\frac{\left(K\left(1\left(\omega_{1} t\right) t^{\prime}\right)\right)_{11}}{\sqrt{\left[1+\left(K^{2}\left(1\left(\omega_{1} t\right) t^{\prime}\right)\right\}_{11}\right]}} .
$$

The specific values for $K$ can be read from eq. (27); for a discussion of the choice of + sign, see appendix $B$.

The $\mathrm{SO}(5) \supset \mathrm{U}(2)$ reduced Wigner coefficients for the coupling with the 10 dimensional representation, $\left(\omega_{1} t\right) \times(11) \rightarrow\left(\omega_{1}^{\prime} t^{\prime}\right)$, follow from the same methods for those representations $\left(\omega_{1}^{\prime} t^{\prime}\right)$ for which the coupling is free of multiplicity, i.e. for $\left(\omega_{1}^{\prime} t^{\prime}\right)=\left(\omega_{1} \pm 1, t^{\prime}\right)$ with $t^{\prime}=t \pm 1, t$ and for $\left(\omega_{1}^{\prime} t^{\prime}\right)=\left(\omega_{1} t^{\prime}\right)$ with $t^{\prime}=t \pm 1$. Results for these cases are collected in table 6 a.

The coupling $\left(\omega_{1} t\right) \times(11) \rightarrow\left(\omega_{1} t\right)$ has a two-fold multiplicity and the Wigner coefficients will be tagged with a subscript $\rho=1$ or 2 . The canonical choice identifies the $\rho=1$ Wigner coefficients with the matrix elements of the generators, (except for a normalization factor which is given by the quadratic $\mathrm{SO}(5)$ Casimir invariant). There remains the task of calculating the Wigner coefficients for $\rho=2$. This is now complicated by the fact that the general 10-dimensional irreducible tensor $T^{(11)}$ given by bifermion operators coupled to even $J\left(J_{\mathrm{e}} \neq 0\right)$, as listed in table 1 , will in general be specified in terms of two $\mathrm{SO}(5)$-reduced matrix elements, the double caret quantities of sect. 3 , one for the $\rho=1$ coupling and another for the $\rho=2$ coupling. The method outlined in sect. 3 can, however, still be used if the ratio of these double-caret reduced matrix elements can be determined first. In this case, e.g.,

$$
\begin{aligned}
& \left\langle p^{\prime}=p-1\left(\omega_{1} t\right) T^{\prime} i^{\prime} ; \alpha^{\prime} J^{\prime}\left\|\sqrt{2}[a \times a]^{J_{\mathrm{c}}}\right\| p\left(\omega_{1} t\right) T i ; \alpha J\right\rangle \\
& =\sum_{p=1}^{2}\left\langle\left(\omega_{1} t\right) H_{1} T i ;(11)-11 \|\left(\omega_{1} t\right) H_{1}^{\prime} T^{\prime} i^{\prime}\right\rangle_{p}\left\langle\left\langle\left(\omega_{1} t\right) \alpha^{\prime} J^{\prime}\left\|T^{(11)}\left(J_{e}\right)\right\|\left(\omega_{1} t\right) \alpha J\right\rangle\right\rangle_{p} \\
& =\sum_{T_{p}, T_{p}^{\prime}}\left(K^{-1}\left(p^{\prime}\left(\omega_{1} t\right) T^{\prime}\right)\right)_{i^{\prime} T_{p}^{\prime}}\left(K\left(p\left(\omega_{1} t\right) T\right)\right)_{T_{p}, i} \\
& \times \sum_{t^{\prime \prime}} U\left(1 t T T_{p}^{\prime} ; t^{\prime \prime} T^{\prime}\right) U\left(t 1 T T_{p}^{\prime} ; t^{\prime \prime} T_{p}\right) \sqrt{\frac{(2 T+1)(2 t+1)}{\left(2 T^{\prime}+1\right)\left(2 t^{\prime \prime}+1\right)}} \\
& \times \frac{1}{\left(K^{2}\left(1\left(\omega_{1} t\right) t^{\prime \prime}\right)\right)_{11}} \sqrt{p}\left\langle(p-1,0) T_{p}^{\prime} ;(10) 1 \|(p 0) T_{p}\right\rangle \\
& \times\left\{(-1)^{t+1-t^{\prime \prime}} \sqrt{\frac{\left(2 t^{\prime \prime}+1\right)}{(2 t+1)}}\left\langle\left(\omega_{1} t\right) \alpha^{\prime} J^{\prime}\left\|\left[a^{\dagger} \times a\right]^{J} \mathrm{c}^{0}\right\|\left(\omega_{1} t\right) \alpha J\right\rangle\right.
\end{aligned}
$$

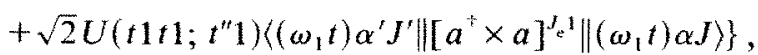

where the reduction of the matrix element of $\sqrt{\frac{1}{2}}\lfloor a \times a\rfloor^{J_{6}^{1}}$ between states of arbitrary 
TABLE 6a

Table of $\mathrm{SO}(5)>\mathrm{U}(2)$ Wigner coefficients for the coupling $\left(\omega_{1} t\right) \times(11) \rightarrow\left(\omega_{1}^{\prime} t^{\prime}\right)$; multiplicity-free cases.

$$
\begin{aligned}
& \left\langle\left(\omega_{1} t\right) H_{1} T i ;(11) h_{1} \tau \|\left(\omega_{1}^{\prime} t^{\prime}\right) H_{1}^{\prime}=H_{1}+h_{1} T^{\prime} i^{\prime}\right\rangle \\
& \quad=\sum_{T_{p}, T_{p}^{\prime}}\left(K^{-1}\left(p^{\prime}\left(\omega_{1}^{\prime} t^{\prime}\right) T^{\prime}\right)\right)_{i^{\prime} T_{p}^{\prime}}\left(K\left(p\left(\omega_{1} t\right) T\right)\right)_{T_{p},} F\left(h_{1} \tau ; T T_{p} ; T^{\prime} T_{p}^{\prime}\right)
\end{aligned}
$$

\begin{tabular}{|c|c|c|c|}
\hline$h_{1}$ & $\tau$ & $p^{\prime}$ & $F\left(h_{1} \tau ; T T_{p} ; T^{\prime} T_{p}^{\prime}\right)$ \\
\hline-1 & 1 & $p$ & $(-1)^{T-T^{\prime}+t^{\prime}-r} U\left(1 t T^{\prime} T_{p} ; t^{\prime} T\right) \delta_{T_{r} T_{p}^{\prime}}$ \\
\hline 0 & 0 & $p+1$ & 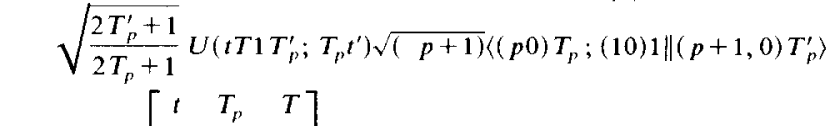 \\
\hline 0 & 1 & $p+1$ & $\sqrt{2}\left[\begin{array}{ccc}1 & 1 & 1 \\
t^{\prime} & T_{p}^{\prime} & T^{\prime}\end{array}\right] \sqrt{(p+1)}\left\langle(p 0) T_{p} ;(10) 1 \|(p+1,0) T_{p}^{\prime}\right\rangle$ \\
\hline+1 & 1 & $p+2$ & $\begin{array}{l}\sqrt{\frac{5}{3}}\left[\begin{array}{ccc}1 & 2 & 1 \\
t^{\prime} & T_{p}^{\prime} & T^{\prime}\end{array}\right] \sqrt{(p+1)(p+2)}\left((p 0) T_{p} ;(20) 2 \|(p+2,0) T_{p}^{\prime}\right\rangle \\
\left.-\frac{1}{2 \sqrt{3}}\left[\begin{array}{ccc}t & T_{p} & T \\
1 & 0 & 1 \\
t^{\prime} & T_{p}^{\prime} & T^{\prime}\end{array}\right] \sqrt{(p+1)(p+2)\left\langle(p 0) T_{p} ;(20) 0 \|(p+2,0) T_{p}^{\prime}\right\rangle}\right\}\end{array}$ \\
\hline
\end{tabular}

Case 1. $\left(\omega_{1}^{\prime} t^{\prime}\right)=\left(\omega_{1}+1 t^{\prime}\right) ; v^{\prime}=v-2$

Case 2. $\left(\omega_{1}^{\prime} t^{\prime}\right)=\left(\omega_{1} t^{\prime}\right) t^{\prime} \neq t, t^{\prime}=t \pm 1 ; v^{\prime}=v$

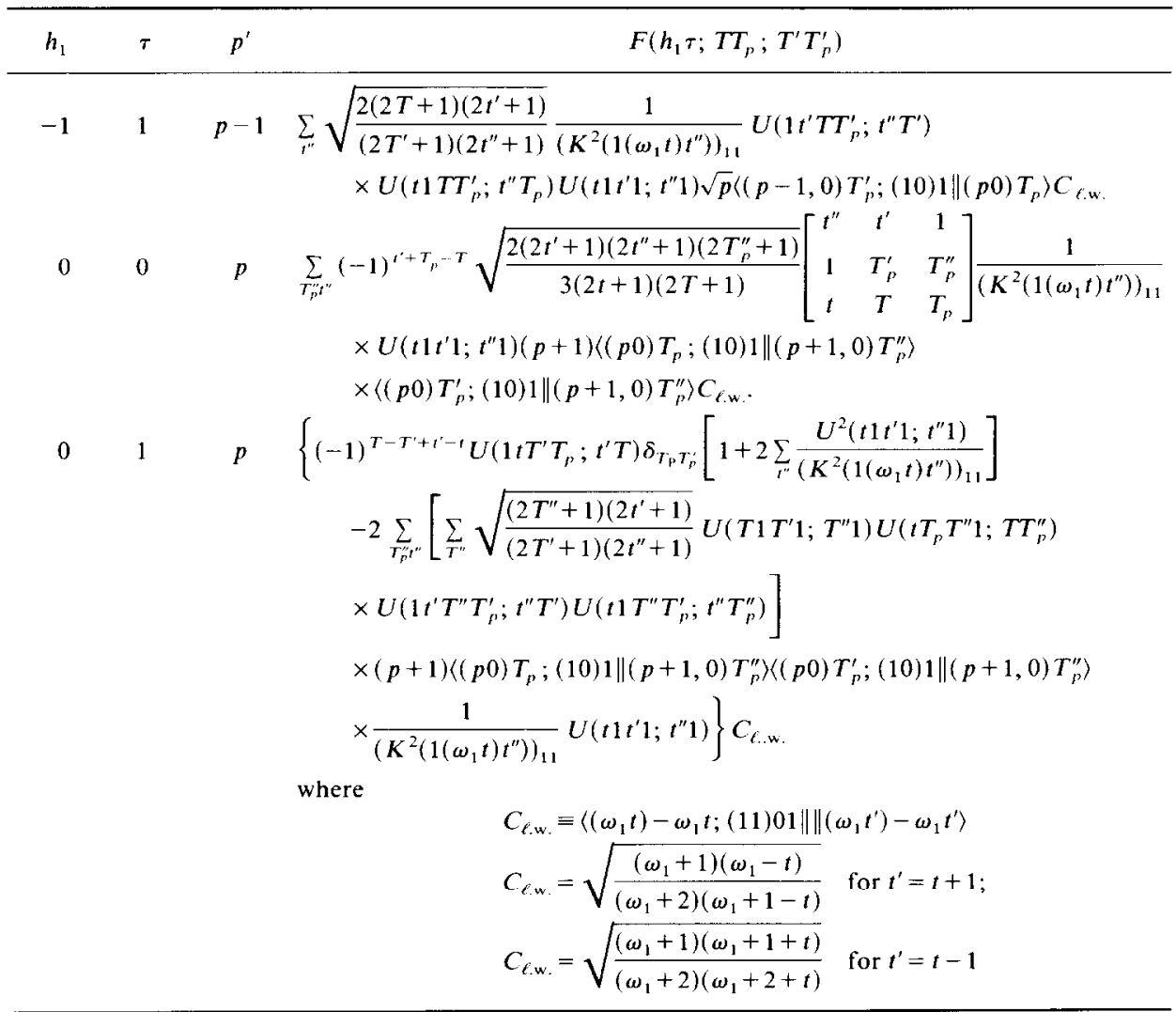


TABLE 6b

Table of $\mathrm{SO}(5) \supset \mathrm{U}(2)$ Wigner coefficients for the coupling $\left(\omega_{1} t\right) \times(11) \rightarrow\left(\omega_{1} t\right)$ with $\rho=2$

$$
\begin{aligned}
& \left\langle\left(\omega_{1} t\right) H_{1} T i(11) h_{1} \tau \|\left(\omega_{1} t\right) H_{1}^{\prime}=H_{1}+h_{1} T^{*} i^{\prime}\right\rangle_{p=?} \\
& =\sum_{T_{p^{n}}, T_{n}^{\prime}}\left(K^{-1}\left(p^{\prime}\left(\omega_{1} t\right) T^{\prime}\right)\right)_{i T_{p}}\left(K\left(p\left(\omega_{1} t\right) T\right)\right)_{r_{r}} \frac{\sqrt{\left[\omega_{1}\left(\omega_{1}+3\right)+t(t+1)\right]}}{\omega_{1}} \\
& \times \sqrt{\frac{\left(\omega_{1}-t\right)\left(\omega_{1}+1\right)\left(\omega_{1}+t+1\right)}{\left(\omega_{1}+2\right)\left(\omega_{1}+2-t\right)\left(\omega_{1}+t+3\right)}} \\
& \times F\left(h_{i} \tau ; T T_{p} ; T^{\prime} T_{p}^{\prime}\right)
\end{aligned}
$$

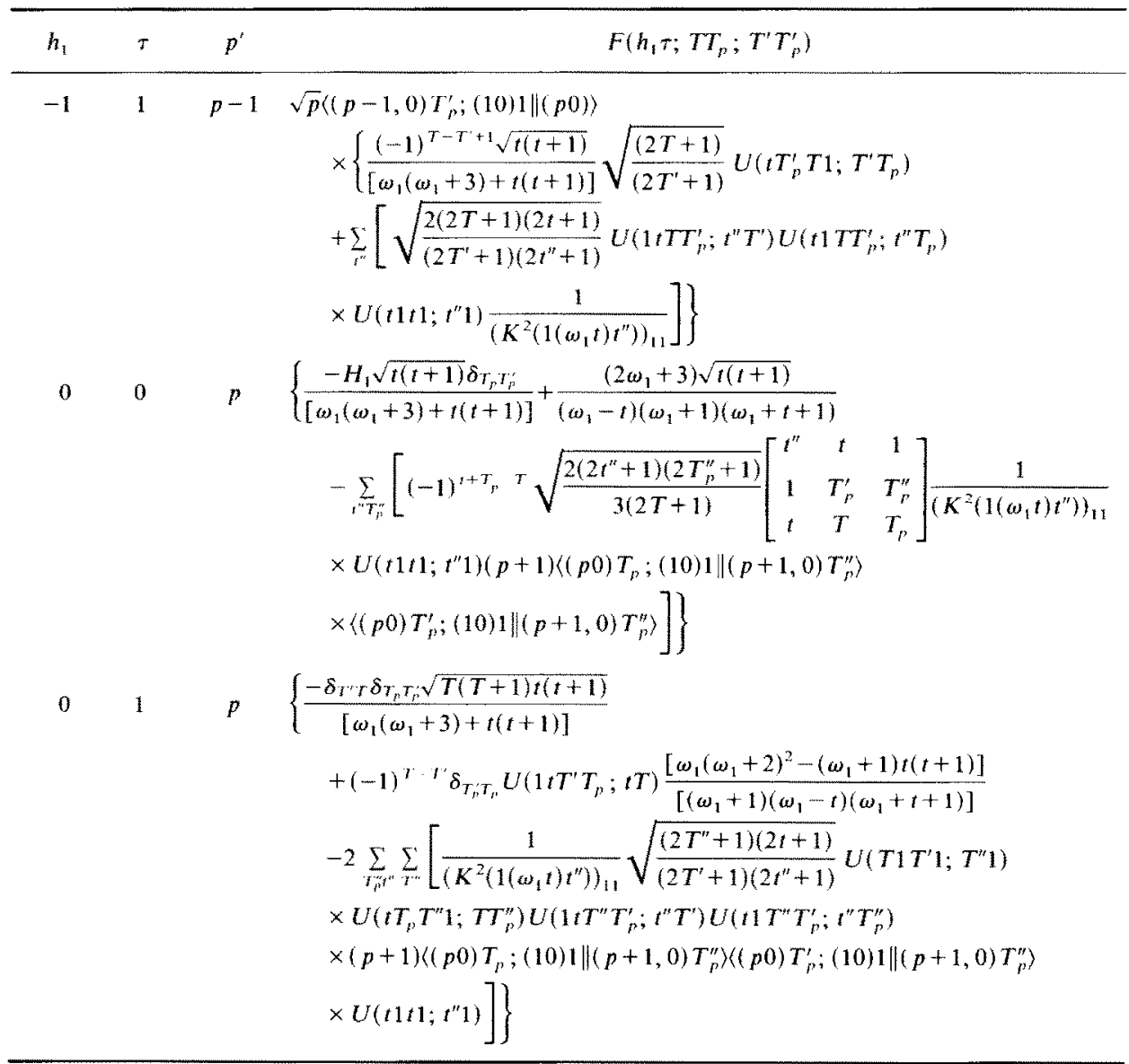


$p, T$ to matrix elements of the intrinsic operator $\sqrt{\frac{1}{2}}[\llbracket \times \backsim]^{J_{\mathrm{e}} 1}$ between starting states has been carried out by recoupling techniques similar to those used in eqs. (50)-(52); and the matrix element of $\sqrt{\frac{1}{2}}[\backsim \times \square]^{J_{\mathrm{e}} 1}$ has been converted to matrix elements in standard representation between purely intrinsic states by the use of entry 9 of table 3. The latter are now given in terms of two $\mathrm{SO}(5)$ Wigner coefficients between lowest weight states and two double caret reduced matrix elements with $\rho=1$ and 2 .

$$
\begin{aligned}
\left\langle\left(\omega_{1} t\right) \alpha^{\prime} J^{\prime}\left\|\left[a^{\dagger} \times a\right]^{J_{\mathrm{e}} 0}\right\|\left(\omega_{1} t\right) \alpha J\right\rangle= & -\sum_{\rho-1}^{2}\left\langle\left(\omega_{1} t\right)-\omega_{1} t ;(11) 00 \|\left(\omega_{1} t\right)-\omega_{1} t\right\rangle_{\rho} \\
& \times\left\langle\left\langle\left(\omega_{1} t\right) \alpha^{\prime} J^{\prime}\left\|T^{(11)}\left(J_{\mathrm{e}}\right)\right\|\left(\omega_{1} t\right) \alpha J\right\rangle\right\rangle_{\rho}, \\
\left\langle\left(\omega_{1} t\right) \alpha^{\prime} J^{\prime}\left\|\left[a^{\dagger} \times a\right]^{J_{\mathrm{c}} 1}\right\|\left(\omega_{1} t\right) \alpha J\right\rangle= & \sum_{\rho=1}^{2}\left\langle\left(\omega_{1} t\right)-\omega_{1} t ;(11) 01 \|\left(\omega_{1} t\right)-\omega_{1} t\right\rangle_{\rho} \\
& \times\left\langle\left(\omega_{1} t\right) \alpha^{\prime} J^{\prime}\left\|T^{(11)}\left(J_{\mathrm{e}}\right)\right\|\left(\omega_{1} t\right) \alpha J\right\rangle_{\rho} .
\end{aligned}
$$

The $\mathrm{SO}(5)$ Wigner coefficients with $\rho-1$ are known from the matrix elements of the $\mathrm{SO}(5)$ generators. Eq. (57) and similar equations for the remaining $T^{(11)}$ irreducible tensor components could now be used with special choices [such as $p^{\prime}=0$, $T_{n}^{\prime}=0$ in eq. (57)], to first calculate both the leading $\mathrm{SO}(5)$ Wigner coefficients needed in eqs. (58) and the ratio of double caret reduced matrix elements. In actual practice it has proved more economical to use another method. The leading $\mathrm{SO}(5)$ Wigner coefficients, with $H_{1}^{\prime}=-\omega_{1} T^{\prime}=t$, are first calculated from the very simple known Wigner coefficients of table 4 through the "buildup" process ${ }^{10}$ )

$$
\begin{aligned}
\sum_{\rho=1}^{2}\left\langle\left(\omega_{1} t\right) h_{1} \tau_{1} ;(11) h_{2} \tau_{2} \|\left(\omega_{1} t\right)-\omega_{1} t\right\rangle_{\rho} U\left(\left(\omega_{1} t\right)\left(\frac{1}{2} \frac{1}{2}\right)\left(\omega_{1} t\right)\left(\frac{1}{2} \frac{1}{2}\right) ;\left(\omega_{1}^{\prime} t^{\prime}\right)_{--} ;(11)_{-} \rho\right) \\
=\sum_{h= \pm \frac{1}{2} \tau^{\prime \prime}}\left\langle\left(\omega_{1} t\right) h_{1} \tau_{1} ;\left(\frac{1}{2} \frac{1}{2}\right) h \frac{1}{2} \|\left(\omega_{1}^{\prime} t^{\prime}\right) h_{1}+h \tau^{\prime \prime}\right\rangle \\
\quad \times\left\langle\left(\omega_{1}^{\prime} t^{\prime}\right) h_{1}+h \tau^{\prime \prime} ;\left(\frac{11}{2} \frac{1}{2}\right) h_{2}-h_{2} \frac{1}{2} \|\left(\omega_{1} t\right)-\omega_{1} t\right\rangle \\
\quad \times\left\langle\left(\frac{11}{2}\right) h \frac{1}{2} ;\left(\frac{11}{2}\right) h_{2}-h \frac{1}{2} \|(11) h_{2} \tau_{2}\right\rangle U\left(\tau_{12} \frac{1}{2} t_{2} ; \tau^{\prime \prime} \tau_{2}\right)
\end{aligned}
$$

Here the $\rho$-dependent U-coefficients are SO(5) Racah coefficients which, for any convenient choice of $\left(\omega_{1}^{\prime} t^{\prime}\right)$, serve merely as normalization factors. Since the Wigner coefficients for $\rho=1$ are known, eq. (59) together with the orthonormality relation

$$
\begin{aligned}
\sum_{h_{1} \tau_{1}} \sum_{h_{2} \tau_{2}}\left\langle\left(\omega_{1} t\right) h_{1} \tau_{1} ;(11) h_{2} \tau_{2} \|\left(\omega_{1} t\right)-\omega_{1} t\right\rangle_{\rho} \\
\quad \times\left\langle\left(\omega_{1} t\right) h_{1} \tau_{1} ;(11) h_{2} \tau_{2} \|\left(\omega_{1} t\right)-\omega_{1} t\right\rangle_{\rho^{\prime}}=\delta_{\rho \rho^{\prime}},
\end{aligned}
$$

can be used to calculate the two U-coefficients with $\rho=1$ and $\rho=2$ and the wanted Wigner coefficients with $\rho=2$. Results are collected in table 7. With these results 
eq. (57) with the special choice $p=1 ; p^{t}=0, T_{p}^{\prime}=0, T=t \pm 1$, or $t$, leads to

$$
\begin{aligned}
& \sum_{\rho=1}^{2}\left\langle\left(\omega_{1} t\right)-\omega_{1}+1 T ;(11)-11 \|\left(\omega_{1} t\right)-\omega_{1} t\right\rangle_{\rho}\left\langle\left(\omega_{1} t\right) \alpha^{\prime} J^{\prime}\left\|T^{(1 \prime \prime}\left(J_{\mathrm{e}}\right)\right\|\left(\omega_{1} t\right) \alpha J\right\rangle_{\rho} \\
&= \frac{1}{\left(K\left(1\left(\omega_{1} t\right) T\right)\right)_{11}}(-1)^{t-T} \sqrt{\frac{(2 T+1)}{(2 t+1)}}\left\{\sum_{\rho=1}^{2}\left\langle\left(\omega_{1} t\right)-\omega_{1} t ;(11) 00 \|\left(\omega_{1} t\right)-\omega_{1} t\right\rangle_{p}\right. \\
& \times\left\langle\left\langle\left(\omega_{1} t\right) \alpha^{\prime} J^{\prime}\left\|T^{\prime \prime \prime}\left(J_{\mathrm{e}}\right)\right\|\left(\omega_{1} t\right) \alpha J\right\rangle_{\rho}+\frac{1}{2 \sqrt{t(t+1)}}[2+t(t+1)-T(T+1)]\right. \\
& \times \sum_{\rho=1}^{2}\left\langle\left(\omega_{1} t\right)-\omega_{1} t ;(11) 01 \|\left(\omega_{1} t\right)-\omega_{1} t\right\rangle_{p}\left\langle\left\langle\left(\omega_{1} t\right) \alpha^{\prime} J^{\prime}\left\|T^{(11)} J_{\mathrm{e}}\right\|\left(\omega_{1} t\right) \alpha J\right\rangle_{\rho}\right\} .
\end{aligned}
$$

With the entries of table 7 this leads to

$$
\frac{\left\langle\left\langle\alpha^{\prime} J^{\prime}\left\|T^{(11)}\left(J_{\mathrm{e}}\right)\right\|\left(\omega_{1} t\right) \alpha J\right\rangle_{\beta=1}\right.}{\left\langle\left\langle\alpha^{\prime} J^{\prime}\left\|T^{(11)}\left(J_{\mathrm{e}}\right)\right\|\left(\omega_{1} t\right) \alpha J\right\rangle_{x=2}\right.}=\frac{\sqrt{t(t+1)}}{\omega_{1}} \sqrt{\frac{\left(\omega_{1}+1\right)\left(\omega_{1}-t\right)\left(\omega_{1}+t+1\right)}{\left(\omega_{1}+2\right)\left(\omega_{1}+2-t\right)\left(\omega_{1}+3+t\right)}}
$$

and

\begin{tabular}{|c|c|c|c|c|c|}
\hline$h_{1}$ & $\tau_{1}$ & $h_{2}$ & $\tau_{2}$ & $\rho=1$ & $\rho=2$ \\
\hline$-\omega_{1}$ & $t$ & 0 & 0 & $-\omega_{1} N_{1}$ & $\sqrt{t(t+1)} N_{2}$ \\
\hline$-w_{1}$ & $t$ & 0 & 1 & $\sqrt{t}(t+1) N_{1}$ & $\left(\omega_{1}+3\right) N_{2}$ \\
\hline$-w_{1}+1$ & $t+1$ & -1 & 1 & $\sqrt{\frac{(2 t+3)\left(\omega_{1}-t\right)}{(2 t+1)}} N_{t}$ & $\left(\omega_{1}+4+t\right) \sqrt{\frac{t(2 t+3)}{(t+1)(2 t+1)\left(\omega_{1}-t\right)}}$ \\
\hline$-\omega_{1}+1$ & $t-1$ & -1 & 1 & $-\sqrt{\frac{(2 t-1)\left(\omega_{1}+1+t\right)}{(2 t+1)}} N_{1}$ & $-\left(\omega_{1}+3-t\right) \sqrt{\frac{(2 t-1)(t+1)}{(2 t+1) t\left(\omega_{1}+t+1\right)}}$ \\
\hline$-\omega_{1}+1$ & $t$ & -1 & 1 & $\sqrt{\left(\omega_{1}+1\right)} N_{1}$ & $\frac{\left[\left(\omega_{1}+3\right)-t(t+1)\right]}{\sqrt{t(t+1)\left(\omega_{1}+1\right)}} N_{2}$ \\
\hline
\end{tabular}

$$
\begin{gathered}
\sum_{p=1}^{2}\left\langle\left(\omega_{1} t\right)-\omega_{1} t ;(11) 00 \|\left(\omega_{1} t\right)-\omega_{1} t\right\rangle_{p}\left\langle\left(\omega_{1} t\right) \alpha^{\prime} J^{\prime}\left\|T^{(1)}\left(J_{\mathrm{e}}\right)\right\|\left(\omega_{1} t\right) \alpha J\right\rangle_{p}=0 \\
\sum_{p=1}^{2}\left\langle\left(\omega_{1} t\right)-\omega_{1} t ;(11) 01 \|\left(\omega_{1} t\right)-\omega_{1} t\right\rangle_{p}\left\langle\left(\omega_{1} t\right) \alpha^{\prime} J^{\prime}\left\|T^{(11)}\left(J_{\mathrm{e}}\right)\right\|\left(\omega_{1} t\right) \alpha J\right\rangle_{p} \\
=\frac{\sqrt{\omega_{1}\left(\omega_{1}+3\right)+t(t+1)}}{\omega_{1}} \sqrt{\frac{\left(\omega_{1}+1\right)\left(\omega_{1}-t\right)\left(\omega_{1}+t+1\right)}{\left(\omega_{1}+2\right)\left(\omega_{1}+2-t\right)\left(\omega_{1}+t+3\right)}} \\
\quad \times\left\langle\left\langle\left(\omega_{1} t\right) \alpha^{\prime} J^{\prime}\left\|T^{(1)}\left(J_{\mathrm{c}}\right)\right\|\left(\omega_{1} t\right) \|\left(\omega_{1} t\right) \alpha J\right\rangle_{\rho-2} .\right.
\end{gathered}
$$

TABLE 7

Leading SO(5) Wigner coefficients for $\left(\omega_{1} t\right) \times(11) \rightarrow\left(\omega_{1} t\right)\left\langle\left(\omega_{1} t\right) h_{1} \tau_{1} ;(11) h_{2} \tau_{2} \|\left(\omega_{1} t\right)-\omega_{1} t\right\rangle_{\beta}$

$$
\begin{aligned}
& N_{1}=\frac{1}{\sqrt{\left[\omega_{1}\left(\omega_{1}+3\right)+t(t+1)\right]}}, \\
& N_{2}=\sqrt{\frac{\left(\omega_{1}-t\right)\left(\omega_{1}+1\right)\left(\omega_{1}+t+1\right)}{\left(\omega_{1}+2\right)\left(\omega_{1}+2-t\right)\left(\omega_{1}+3+t\right)\left[\omega_{1}\left(\omega_{1}+3\right)+t(t+1)\right]}} .
\end{aligned}
$$


With the results of eqs. (62) and (63), the double-caret matrix element $\left\langle\left(\omega_{1} t\right) \alpha^{\prime} J^{\prime}\left\|T^{(1)}\left(J_{e}\right)\right\|\left(\omega_{1} t\right) \alpha J\right\rangle_{p=2}$ will drop out of eq. (57); and this equation, together with the analogous equations for the remaining (11)-tensor components, can be solved for the needed Wigner coefficients with $\rho=2$. These are collected in table $6 \mathrm{~b}$. Coefficients which can be obtained from tabulated coefficients via simple symmetry properties are again omitted for brevity. The last entry in table $6 \mathrm{~b}$ involves a sum over $T^{\prime \prime}$ of a product of four Racah coefficients which could have been abbreviated in terms of a $12-j$ symbol.

The Wigner coefficients with $\rho=1$ follow, with a normalization factor of $\left[\omega_{1}\left(\omega_{1}+\right.\right.$ $3)+t(t+1)]^{1 / 2}$, from the matrix elements of the $\mathrm{SO}(5)$ generators. Eqs. (17)-(19) lead to

$$
\begin{aligned}
\left\langle\left(\omega_{1} t\right)\right. & \left.H_{1} T i ;(11)+11 \|\left(\omega_{1} t\right) H_{1}+1 T^{\prime} i^{\prime}\right\rangle_{p=1} \\
= & \sum_{T_{p}^{\prime}, T_{p}}\left(K\left(p+1\left(\omega_{1} t\right) T^{\prime}\right)\right)_{T_{p}^{\prime} r^{\prime}}\left(K^{-1}\left(p\left(\omega_{1} t\right) T\right)\right)_{i T_{p}} \frac{1}{\sqrt{\left[\omega_{1}\left(\omega_{1}+3\right)+t(t+1)\right]}} \\
& \times U\left(t T_{p} T^{\prime} 1 ; T T_{p}^{\prime}\right) \sqrt{(p+1)}\left\langle(p 0) T_{p} ;(10) 1 \|(p+1,0) T_{p}^{\prime}\right\rangle .
\end{aligned}
$$

Similarly, eqs. (9a) and (10) directly give

$$
\begin{aligned}
\left\langle\left(\omega_{1} t\right) H_{1} T i ;(11)-11 \|\left(\omega_{1} t\right) H_{1}-1 T^{\prime} i^{\prime}\right\rangle_{p=1} \\
=\sum_{T_{p}^{\prime}, T_{p}}\left(K^{-1}\left(p-1\left(\omega_{1} t\right) T^{\prime}\right)\right)_{i^{\prime} T_{p}^{\prime}}\left(K\left(p\left(\omega_{1} t\right) T\right)\right)_{T_{r^{\prime}} i} \frac{1}{\sqrt{\left[\omega_{1}\left(\omega_{1}+3\right)+t(t+1)\right]}} \\
\quad \times(-1)^{T-T^{\prime}} \sqrt{\frac{(2 T+1)}{\left(2 T^{\prime}+1\right)}} U\left(t T_{p}^{\prime} T 1 ; T^{\prime} T_{p}\right) \sqrt{p}\left\langle(p-1,0) T_{p}^{\prime} ;(10) 1 \|(p 0) T_{p}\right\rangle .
\end{aligned}
$$

Finally,

$$
\begin{gathered}
\left\langle\left(\omega_{1} t\right) H_{1} T i ;(11) 00 \|\left(\omega_{1} t\right) H_{1} T i^{\prime}\right\rangle_{\rho=1}=\frac{H_{1}}{\sqrt{\left[\omega_{1}\left(\omega_{1}+3\right)+t(t+1)\right]}} \delta_{i i^{\prime}}, \\
\left\langle\left(\omega_{1} t\right) H_{1} T i ;(11) 01 \|\left(\omega_{1} t\right) H_{1} T^{\prime} i^{\prime}\right\rangle_{\rho=1}=\frac{\sqrt{T(T+1)}}{\sqrt{\left[\omega_{1}\left(\omega_{1}+3\right)+t(t+1)\right]}} \delta_{i i}^{\prime} \delta_{T T^{\prime}} .
\end{gathered}
$$

\section{Appendix A}

\section{THE $K$-MATRIX ELEMENTS}

The $K$-matrix elements which form a vital part of this investigation follow from eqs. (23), (24), and the knowledge of the hermitian (real) $\left(K K^{\dagger}\right)$ matrices. Recall that these are diagonal in $\left(\omega_{1} t\right), p$, and $T$. Their dimension is given by the number of possible $T_{p}$ values for a given $p$ and $T$. One-dimensional $\left(K K^{\dagger}\right)$ matrix elements, (with $K=K^{\dagger}$ ), follow at once from a recursive application of eq. (25). In the general case, the $\left(K K^{\dagger}\right)_{T_{n} \tau_{p}}$ can be evaluated through recursion relations which follow from eqs. (11) and (12),

$$
K K^{\dagger} z=\left(A_{\mathrm{op}} z-z A_{\mathrm{op}}\right) K K^{\dagger}
$$


by taking matrix elements between states with fixed $p, T_{p}, T$ on the right and fixed $p+1, T_{p}^{\prime}, T^{\prime}$ on the left. This leads to the recursion relation

$$
\begin{aligned}
\sum_{\bar{T}_{p}^{\prime}}\left(K K^{\dagger}\left(p+1\left(\omega_{1} t\right) T^{\prime}\right)\right)_{T_{p} \bar{T}_{p}} U\left(t T_{p} T^{\prime} 1 ; T \bar{T}_{p}^{\prime}\right) \sqrt{p+1}\left\langle(p 0) T_{p} ;(10) 1 \|(p+1,0) \bar{T}_{p}^{\prime}\right\rangle \\
=\sum_{\bar{T}_{p}}\left(A_{p+1} T_{p}^{\prime} T^{\prime}-\Lambda_{p} \bar{T}_{p} r\right) U\left(t \bar{T}_{p} T^{\prime} 1 ; T T_{p}^{\prime}\right) \\
\quad \times \sqrt{p+1}\left((p 0) \bar{T}_{p} ;(10) 1 \|(p+1,0) T_{p}^{\prime}\right\rangle\left(K K^{\dagger}\left(p\left(\omega_{1} t\right) T\right)\right)_{\bar{T}_{p} T_{p},}
\end{aligned}
$$

For numerical calculations it may be more efficient to take the scalar product of the $z$-vector eq. (A.1) with the $\nabla$ operator,

$$
K K^{\dagger}(z \cdot \nabla)=\sum_{i}\left(\Lambda_{\text {op }} z_{i}-z_{i} \Lambda_{\text {op }}\right) K K^{\dagger} \nabla_{i}
$$

This leads to the expression of a single $\left(K K^{*}\left(p+1\left(\omega_{1} t\right) T^{\prime}\right)\right)$ matrix element in terms of $\left(K K^{\dagger}\left(p\left(\omega_{1} t\right) T\right)\right)$ matrix elements, all of which are known from the previous step in the process.

$$
\begin{aligned}
\left(K K^{\dagger}\left(p+1\left(\omega_{1} t\right) T^{\prime}\right)\right)_{T_{p}^{\prime} \bar{T}_{p}^{\prime}=} & \frac{1}{(p+1)} \sum_{T} \sum_{T_{p} \bar{T}_{p}}\left(A_{p+1} T_{p}^{\prime} T^{\prime}-A_{p} \bar{T}_{p}\right) \\
& \times\left(K K^{\dagger}\left(p\left(\omega_{1} t\right) T\right)\right)_{\bar{T}_{p} T_{p}} U\left(t T_{p} T^{\prime} 1 ; T \bar{T}_{p}^{\prime}\right) U\left(t \bar{T}_{p} T^{\prime} 1 ; T T_{p}^{\prime}\right) \\
& \times(p+1)\left\langle(p 0) \bar{T}_{p} ;(10) 1 \|(p+1,0) T_{p}^{\prime}\right\rangle \\
& \times\left\langle(p 0) T_{p} ;(10) 1 \|(p+1,0) \bar{T}_{p}^{\prime}\right\rangle .
\end{aligned}
$$

However, a judicious use of the simpler recursion relation, eq. (A.2), makes it possible to give analytic formulae for almost all states of practical interest in shell-model applications. These include all states in irreducible representations $\left(\omega_{1} t\right)$, with $t \leqslant \frac{3}{2}$, and all states with $p \leqslant 4$ for arbitrary $\left(\omega_{1} t\right)$. Recall that $\omega_{1}=j+\frac{1}{2}-\frac{1}{2} v$, (or $\omega_{1}=\sum\left(j+\frac{1}{2}\right)-\frac{1}{2} v$ for mixed configurations); $v=$ seniority number.

The $K K^{+}=K^{2}$ matrices for all states in irreducible representations $\left(\omega_{1} 0\right)$ and $\left(\omega_{12}\right)$ are 1 -dimensional and are given by

1. $\left(\omega_{1} 0\right)$

$$
\left(K^{2}\left(p\left(\omega_{1} 0\right) T\right)\right)_{T T}=\frac{\omega_{1} !\left(2 \omega_{1}+1\right) ! !}{\left(\omega_{1}-\frac{1}{2}(p+T)\right) ! 2^{\frac{1}{2(p-T)}}\left(2 \omega_{1}+1-p+T\right) ! !}
$$

Note that $T_{p}=T$ in this case.

2. $\left(\omega_{1} \frac{1}{2}\right)$

There are two cases

$$
\begin{aligned}
& \left(K^{2}\left(p\left(\omega_{1} \frac{1}{2}\right) T=T_{p}+\frac{1}{2}\right)\right)_{T_{p} T_{p}}=\frac{\left(\omega_{1}-\frac{1}{2}\right) !\left(2 \omega_{1}+2\right) ! !}{\left(\omega_{1}-\frac{1}{2}-\frac{1}{2}\left(p+T_{p}\right)\right) ! 2^{!\left(p-T_{p}\right)}\left(2 \omega_{1}+2-p+T_{p}\right) ! !}, \\
& \left(K^{2}\left(p\left(\omega_{1} \frac{1}{2}\right) T=T_{p}-\frac{1}{2}\right)\right)_{T_{n} T_{n}}=\frac{\left(\omega_{1}-\frac{1}{2}\right) !\left(2 \omega_{1}+2\right) ! !}{\left(\omega_{1}+\frac{1}{2}-\frac{1}{2}\left(p+T_{p}\right)\right) ! 2^{\frac{1}{2}\left(p T_{p}\right)+1}\left(2 \omega_{1}-p+T_{p}\right) ! !}
\end{aligned}
$$


Note that $\omega_{1}$ must be a half-integer in this case. The first case with $T=T_{p}+\frac{1}{2}$ was named o-type, while the second case with $T=T_{p}-\frac{1}{2}$ was named e-type in ref. ${ }^{\text {"I }}$ ).

\section{3. $\left(\omega_{1} 1\right)$}

Analytic formulae for this representation were given in ref. $\left.{ }^{9}\right)$, the $\left(K K^{\dagger}(p=T+\right.$ $\left.\left.2 k\left(\omega_{1} 1\right) T=T_{p}\right)\right)_{T_{p} T_{p}}$ are 1-dimensional and are given through eq. (24) of ref. $\left.{ }^{9}\right)$. The $\left(K K^{+}\left(p=T+2 k+1\left(\omega_{1} 1\right) T\right)\right)$ matrices are in general 2-dimensional, with $T_{p}=T \pm 1$, and are given through eqs. (25) of ref. $\left.{ }^{9}\right)$.

4. $\left(\omega_{1} \frac{3}{2}\right)$

There are again two cases, both of them 2-dimensional, in general. In the first case

$$
\begin{gathered}
p=T+\frac{1}{2}+2 k, \quad \text { with } T_{p}=T-\frac{3}{2}, T+\frac{1}{2} ; k=0,1,2, \cdots, \\
\left(K K^{\dagger}\left(p=T+\frac{1}{2}+2 k\left(\omega_{1 \frac{3}{2}}^{3}\right) T\right)\right)_{1_{p} 1_{p}^{\prime}}-\frac{\left(\omega_{1}-\frac{3}{2}\right) ! \Gamma\left(\omega_{1}+2\right)}{4 T\left(\omega_{1}-T-k\right) ! \Gamma\left(\omega_{1}+2-k\right)} M_{T_{p} T_{p}^{\prime}},
\end{gathered}
$$

with

$$
\begin{aligned}
& M_{T-\frac{3}{2}, T-\frac{3}{2}}=\left[4\left(\omega_{1}-T-k\right) T\left(\omega_{1}+2\right)+3(k+1)\right], \\
& M_{T+\frac{1}{2}, T+\frac{1}{2}}=\left[4 T\left(\omega_{1}+1\right)\left(\omega_{1}+1-k\right)-3(T+k+1)\right], \\
& M_{T-\frac{3}{2}, T+\frac{1}{2}}=\sqrt{3(2 T-1)(2 T+3)(k+1)(T+k+1)} .
\end{aligned}
$$

For the second case,

$$
\begin{gathered}
p=T+\frac{1}{2}+2 k+1, \quad \text { with } T_{p}=T+\frac{3}{2}, T-\frac{1}{2} ; k=0,1,2, \ldots, \\
\left(K K^{+}\left(p=T+\frac{1}{2}+2 k+1\left(\omega_{1} \frac{3}{2}\right) T\right)\right)_{T_{n} T_{p}^{\prime}}=\frac{\left(\omega_{1}-\frac{3}{2}\right) ! \Gamma\left(\omega_{1}+2\right)}{4(T+1)\left(\omega_{1}-T-k\right) ! \Gamma\left(\omega_{1}+1-k\right)} M_{T_{r} T_{p}^{\prime},}
\end{gathered}
$$

with

$$
\begin{aligned}
& M_{T+\frac{3}{2}, T+\frac{3}{2}}=\left[4(T+1)\left(\omega_{1}+2\right)\left(\omega_{1}-k\right)-3(T+k+2)\right], \\
& M_{T-\frac{1}{2}, T-\frac{1}{2}}=\left[4\left(\omega_{1}-T-k\right)(T+1)\left(\omega_{1}+1\right)+3(k+1)\right], \\
& M_{T+2, T-\frac{1}{2}}=\sqrt{3(2 T-1)(2 T+3)(k+1)(T+k+2)} .
\end{aligned}
$$

Note that states with $T=\omega_{1}-k$ lie on the periphery of Pauli-allowed values on a $p, T$ diagram and should therefore be "simple", with a single Pauli-allowed occurrence. It can be seeen at once that the above $2 \times 2\left(K K^{\dagger}\right)$-matrices have one zero eigenvalue for $T=\omega_{1}-k$ and hence only a single allowed value, $i$, corresponding to the single nonzero eigenvalue $\lambda_{i}$, in this special case. 
5. States with $p \leqslant 4$, arbitrary $\left(\omega_{1} t\right)$

States with $T=t+p, t+p-1, t-p, t-p-1$ have 1 -dimensional $K^{2}$ matrices, with $T_{p}$ uniquely fixed at $T_{p}=p$. For these

$$
\begin{aligned}
\left(K^{2}\left(p\left(\omega_{1} t\right) T=t+p\right)\right)_{p p} & =\frac{\left(\omega_{1}-t\right) !}{\left(\omega_{1}-t-p\right) !} \\
\left(K^{2}\left(p\left(\omega_{1} t\right) T=t+p-1\right)\right)_{p p} & =\frac{\left(\omega_{1}+1\right)\left(\omega_{1}-t\right) !}{\left(\omega_{1}+1-t-p\right) !} \\
\left(K^{2}\left(p\left(\omega_{1} t\right) T=t-p\right)\right)_{p p} & =\frac{\left(\omega_{1}+t+1\right) !}{\left(\omega_{1}+t+1-p\right) !} \\
\left(K^{2}\left(p\left(\omega_{1} t\right) T=t-p+1\right)\right)_{p p} & =\frac{\left(\omega_{1}+1\right)\left(\omega_{1}+t+1\right) !}{\left(\omega_{1}+2+t-p\right) !}
\end{aligned}
$$

The remaining states, with $p \leqslant 4$, have 2 - or 3-dimensional ( $K K^{*}$ ) matrices, and will be given by

$$
\left(K K^{\dagger}\left(p\left(\omega_{1} t\right) T\right)\right)_{T_{n}, T_{p}^{\prime}}=(\mathrm{C} . \mathrm{F} .) M_{T_{n} T_{p}^{\prime}},
$$

where (C.F.) is a common factor.

$$
\left(K K^{\dagger}\left(2\left(\omega_{1} t\right) t\right)\right)_{T_{n} T_{p}^{\prime}} \quad \text { has }(\mathrm{C} . \mathrm{F} .)=1,
$$

with

$$
\begin{gathered}
M_{00}=\left[{ }_{2}^{1} \omega_{1}\left(2 \omega_{1}+1\right)-\frac{2}{3} t(t+1)\right], \\
M_{22}=\left[\left(\omega_{1}+1\right)^{2}-\frac{1}{3} t(t+1)\right], \\
M_{02}={ }_{3}^{1} \sqrt{{ }_{2}^{1} t(t+1)(2 t-1)(2 t+3)}, \\
\left(K K^{\dot{\prime}}\left(3\left(\omega_{1} t\right) t+1\right)\right)_{T_{p}, T_{p}^{\prime}} \text { has (C.F.) }=\left(\omega_{1}-t\right),
\end{gathered}
$$

with

$$
\begin{gathered}
M_{11}=\left[{ }_{2}^{1}\left(2 \omega_{1}+1\right)\left(\omega_{14}-1\right)-{ }_{5}^{4} t(t+2)\right], \\
M_{33}=\left\lfloor\left(\omega_{1}+1\right)^{2}-{ }_{5}^{1} t(t+2)\right\rfloor, \\
M_{13}=\frac{1}{5} \sqrt{t(t+2)(2 t-1)(2 t+5)}, \\
\left(K K^{\dot{*}}\left(3\left(\omega_{1} t\right) t\right)\right)_{T_{r,} T_{r}^{\prime}} \text { has (C.F.) }=\left(\omega_{1}+1\right),
\end{gathered}
$$

with

$$
\begin{gathered}
M_{11}=\left[1_{2}^{1}\left(2 \omega_{1}+1\right)\left(\omega_{1}-1\right)-{ }_{5}^{2}(t+2)(t-1)\right], \\
M_{33}=\left[\left(\omega_{1}+1\right)^{2}-\frac{1}{5}(3 t(t+1)-1)\right], \\
M_{13}=\frac{1}{5} \sqrt{\frac{3}{2}(t-1)(t+2)(2 t-1)(2 t+3)}, \\
\left(K K^{\dagger}\left(3\left(\omega_{1} t\right) t-1\right)\right)_{T_{r}, T_{r}} \quad \text { has (C.F.) }=\left(\omega_{1}+t+1\right),
\end{gathered}
$$


with

$$
\begin{gathered}
M_{11}=\left[\frac{1}{2}\left(2 \omega_{1}+1\right)\left(\omega_{1}-1\right)-\frac{4}{5}(t+1)(t-1)\right], \\
M_{33}=\left[\left(\omega_{1}+1\right)^{2}-\frac{1}{5}(t+1)(t-1)\right], \\
M_{13}=\frac{1}{5} \sqrt{(t-1)(t+1)(2 t-3)(2 t+3)}, \\
\left(K K^{+}\left(4\left(\omega_{1} t\right) t+2\right)\right)_{T_{p} T_{r}^{\prime}} \text { has (C.F.) }=\left(\omega_{1}-t\right)\left(\omega_{1}-t-1\right),
\end{gathered}
$$

with

$$
\begin{aligned}
& M_{22}=\left[\left(\omega_{1}^{2}-\frac{3}{2} \omega_{1}-1\right)-\frac{6}{7} t(t+3)\right], \\
& M_{44}=\left[\left(\omega_{1}+1\right)^{2}-\frac{1}{7} t(t+3)\right], \\
& M_{24}=\frac{1}{7} \sqrt{\frac{3}{2} t(t+3)(2 t-1)(2 t+7)},
\end{aligned}
$$

$\left(K K^{\dagger}\left(4\left(\omega_{1} t\right) t-2\right)\right)_{T_{n} T_{n}^{\prime}}$ has (C.F.) $=\left(\omega_{1}+t\right)\left(\omega_{1}+t+1\right)$,

with

$$
\begin{gathered}
M_{22}=\left[\left(\omega_{1}^{2}-\frac{3}{2} \omega_{1}-1\right)-\frac{6}{7}(t+1)(t-2)\right], \\
M_{44}=\left[\left(\omega_{1}+1\right)^{2}-\frac{1}{7}(t+1)(t-2)\right], \\
M_{24}=\frac{1}{7} \sqrt{\frac{3}{2}(t+1)(t-2)(2 t-5)(2 t+3)}, \\
\left(K K^{+}\left(4\left(\omega_{1} t\right) t+1\right)\right)_{T_{p} T_{p}^{\prime}} \quad \text { has (C.F.) }=\left(\omega_{1}+1\right)\left(\omega_{1}-t\right),
\end{gathered}
$$

with

$$
\begin{gathered}
M_{22}=\left[\omega_{1}^{2}-\frac{3}{2} \omega_{1}-\frac{1}{7}(2 t+5)(2 t-1)\right], \\
M_{44}=\left[\omega_{1}\left(t_{1}+2\right)-\frac{3}{7}(t-1)(t+3)\right], \\
M_{24}=\frac{1}{7} \sqrt{3(t-1)(t+3)(2 t+5)(2 t-1)}, \\
\left(K K^{\dagger}\left(4\left(\omega_{1} t\right) t-1\right)\right)_{T_{r} T_{r}^{\prime}} \text { has (C.F.) }=\left(\omega_{1}+1\right)\left(\omega_{1}+t+1\right),
\end{gathered}
$$

with

$$
\begin{aligned}
& M_{22}=\left[\omega_{1}^{2}-\frac{3}{2} \omega_{1}-\frac{1}{7}(2 t+3)(2 t-3)\right], \\
& M_{44}=\left[\omega_{1}\left(\omega_{1}+2\right)-\frac{3}{7}(t-2)(t+2)\right], \\
& M_{24}=\frac{1}{7} \sqrt{3(t-2)(t+2)(2 t+3)(2 t-3)} .
\end{aligned}
$$

Finally,

$$
\left(K K^{+}\left(4\left(\omega_{1} t\right) t\right)\right)_{T_{p} T_{p}^{\prime}} \quad \text { has }(\mathrm{C} . \mathrm{F} .)=1
$$


with

$$
\begin{aligned}
M_{00}= & \left\{\left(\omega_{1}-\frac{1}{2}\right)^{2} \omega_{1}^{2}-\frac{1}{3}\left(\omega_{1}-\frac{1}{2}\right) \omega_{1}\left[4 t(t+1)+\frac{3}{2}\right]+\frac{2}{15} t(t+1)(2 t-1)(2 t+3)\right\}, \\
M_{02}= & {\left[7\left(2 \omega_{1}+1\right)\left(\omega_{1}-1\right)-8(t+2)(t-1)\right]_{6}^{1} \sqrt{\frac{1}{35} t(t+1)(2 t-1)(2 t+3)}, } \\
M_{04}= & \frac{1}{5} \sqrt{\frac{1}{14}(t-1) t(t+1)(t+2)(2 t-3)(2 t-1)(2 t+3)(2 t+5)}, \\
M_{22}= & \left\{\frac{1}{7}(2 t-3)(2 t+3)(t-1)(t+1)+\left(\omega_{1}-t\right)\left[\omega_{1}^{2}+\omega_{1}^{2}\left(t+\frac{1}{2}\right)\right.\right. \\
& \left.\left.+\frac{1}{42} \omega_{1}\left(8 t^{2}-13 t-30\right)+\frac{1}{42}\left(8 t^{3}-32 t^{2}-49 t+45\right)\right]\right\}, \\
M_{24}= & {\left[3\left(\omega_{1}+1\right)^{2}-t(t+1)\right] \frac{1}{7} \sqrt{\frac{2}{5}(t-1)(t+2)(2 t-3)(2 t+5)}, } \\
M_{44}= & \left\{\omega_{1}\left(\omega_{1}+1\right)^{2}\left(\omega_{1}+2\right)-\frac{6}{7}\left(\omega_{1}+1\right)^{2}(t+2)(t-1)+\frac{3}{35}(t-1) t(t+1)(t+2)\right\} .
\end{aligned}
$$

More complicated cases can be obtained from these by direct application of eq. (A.4).

\section{Appendix B}

\section{SYMMETRY PROPERTIES}

Symmetry properties of the $\mathrm{SO}(5) \supset \mathrm{U}(2)$ reduced Wigner coefficients depend on the conjugation properties of the $\mathrm{SO}(5)$ states. The group $\mathrm{SO}(5)$ is self-adjoint. If the set of matrices, $D$, for the elements of $\mathrm{SO}(5)$ form an irreducible representation, the complex conjugates of these matrices, $D^{*}$, form an equivalent irreducible representation. The basis vectors of an irreducible representation, $\left(\omega_{1} t\right)$, and their conjugates should thus be simply related. Conjugation converts a state with $H_{1}, T$, $M_{T}$ into a state with $-H_{1}, T,-M_{T}$ and is thus equivalent to particle-hole conjugation. Particle states with $H_{1} \leqslant 0, n \leqslant(2 j+1)$, are converted into hole states with $H_{1} \geqslant 0, n \geqslant 2 j+1$, upon conjugation. In the vector coherent state construction, however, states of arbitrary $n$ have so far been constructed from intrinsic states, with $n=v$, and the addition of $p=\frac{1}{2}(n-v) J=0, T=1$-coupled pairs reaching up to a maximum particle number $n=4 j+2-v$. This leads to a very different structure of possible $T_{p}$ values for hole states with $H_{1} \geqslant 0,(n \geqslant 2 j+1)$, compared with that of the corresponding particle states with $H_{1} \leqslant 0,(n \leqslant 2 j+1)$. As a simple example, in the irreducible representation, $\left(\omega_{1} t\right)=(11)$, the intrinsic state with $n=v, p=0$, or $H_{1}=-1$, is a state with $T_{p}=0$, with 1 -dimensional $\left(K K^{*}\right)$ matrix and $K=1$. Its conjugate partner, with $H_{1}=+1$, is a state with $p=2$ and two possible $T_{p}$ values, $T_{p}=0$ and 2 . The $\left(K K^{+}\right)$matrix, (see eq. (A.14)), is 2 -dimensional and is given by

$$
\left(\begin{array}{cc}
\frac{1}{6} & \frac{1}{2} \sqrt{5} \\
1 & 10 \\
3 & 10
\end{array}\right) \text {. }
$$

However, this matrix has one zero eigenvalue and thus leads to a single allowed state, $i=1$, corresponding to the nonzero eigenvalue, $\lambda_{i}=\frac{21}{6}$. The needed $K$-matrix 
elements for this single allowed state are

$$
\begin{array}{cc}
K_{T_{p}=0, i=1}=\sqrt{\frac{1}{6}}, & K_{T_{p}=2, i=1}=\sqrt{\frac{20}{6}}, \\
\left(K^{-1}\right)_{i=1, T_{p}=0}={ }_{21}^{1} \sqrt{6}, & \left(K^{-1}\right)_{i=1, T_{p}=2}-\frac{1}{21} \sqrt{120} ;
\end{array}
$$

and the single state with $H_{1}=+1$ and $p=2$ is given by

$$
\begin{gathered}
\frac{1}{21} \sqrt{6}\left[Z_{T_{r}=0}^{(20)}\left(\boldsymbol{A}^{\dagger}\right) \times\left|\left(\omega_{1} t\right)=(11)\right\rangle\right]_{T=1 M_{1}}, \\
+\frac{1}{21} \sqrt{120}\left[Z_{T_{p}=2}^{(20)}\left(\boldsymbol{A}^{\dagger}\right) \times\left|\left(\omega_{1} t\right)=(11)\right\rangle\right]_{T=1 M_{I}},
\end{gathered}
$$

in the notation of eqs. (28)-(29). The matrix element of $\boldsymbol{A}^{*}$ connecting the state with $p=1$ to this single allowed state with $p=2$ is given through eqs. (17)-(19), together with eq. (22), by a linear combination of two terms. The simple result

$$
\left\langle p=2(11) T=1, i^{\prime}=1\left\|\boldsymbol{A}^{\dagger}\right\| p=1(11) T=1\right\rangle=\sqrt{2}
$$

can, however, be obtained much more directly through complex conjugation and the matrix element of $\boldsymbol{A}$ connecting the state with $p=1$ to the state with $p=0$ which is the conjugate partner of the $p=2$ state.

To obtain simple $\mathrm{SO}(5)$ symmetry properties the basis states of the irreducible representation, $\left(\omega_{1} t\right)$, will therefore be constructed through the vector coherent state technique by the following recipe.

(I) Particle states with $H_{1} \leqslant 0$ will be constructed from intrinsic states with $n=v$, $H_{1}=-\omega_{1}$, through the action of the raising operators $\boldsymbol{A}^{\dagger}$.

(II) Hole states with $H_{1} \geqslant 0$ will be constructed from the conjugate intrinsic states with $H_{1}=\omega_{1}, n=4 j+2-v$, through the action of the pair annihilation operators $\boldsymbol{A}$. For states with $H_{1} \geqslant 0$, therefore, the states with maximum possible particle number will be used as the vector generalized "vacuum" states. These instrinsic states, with $H_{1}=\omega_{1}$, will be annihilated by the action of $\boldsymbol{A}^{\dagger}$. For the construction of these hole states the $\boldsymbol{A}^{\dagger}$ will therefore be mapped into their $z$-space realizations $\Gamma\left(\boldsymbol{A}^{*}\right)=\boldsymbol{\nabla}$, whereas the operators $\boldsymbol{A}$ which create hole states will be mapped into $z$-space realizations $\Gamma(\boldsymbol{A})$ which are the analogue of eq. $(9 \mathrm{~d})$ with $\omega_{1}$ replaced by $-\omega_{1}$.

With this construction procedure states $\left|\left(\omega_{1} t\right) H_{1} T M_{T} i\right\rangle$ are related to their conjugates $\left|\left(\omega_{1} t\right)-H_{1} T-M_{T}, i^{c}\right\rangle$ through the simple standard angular momentum conjugation phase factors $(-1)^{T-M_{r}}$; and the quantum numbers $i^{c}$ of the conjugate states are put into $1: 1$ correspondence with the quantum numbers $i,(i=i)$. To achieve this correspondence the $\lambda_{i}$ of the hole states must be put into $1: 1$ correspondence with the $\lambda_{i}$ of the particle states and the overall phases of the matrix elements $U_{i T_{i}}$ of eq. (24) must be chosen in the same way for both hole and particle states.

Overall phases of $\mathrm{SO}(5)$ Wigner coefficients will be fixed, in addition, through a generalized Condon-Shortley phase convention. For this purpose the extremal states with $n=v$, or $H_{1}=-\omega_{1}$, i.e. the intrinsic states used in the construction of particle states with $H_{1} \leqslant 0$, will be singled out as preferred states, (rather than the hole states with maximum $\left.H_{1}=+\omega_{1}\right)$. For the $1 \times 2 \rightarrow 3$ coupling, $\left(\omega_{1} t\right)_{1} \times\left(\omega_{1} t\right)_{2} \rightarrow\left(\omega_{1} t\right)_{3}$, the 
$\mathrm{SO}(5) \supset \mathrm{U}(2)$ reduced Wigner coefficients with $\left(H_{1}\right)_{1}=-\left(\omega_{1}\right)_{1},\left(H_{1}\right)_{3}=-\left(\omega_{1}\right)_{3}$ will be singled out, such that

$$
\left\langle\left(\omega_{1} t\right)_{1}-\left(\omega_{1}\right)_{1} t_{1} ;\left(\omega_{1} t\right)_{2}\left(H_{1}\right)_{2}=\left(\omega_{1}\right)_{1}-\left(\omega_{1}\right)_{3},\left(T_{2}\right)_{\max } \|\left(\omega_{1} t\right)_{3}-\left(\omega_{1}\right)_{3} t_{3}\right\rangle_{o}>0 .
$$

Note that for cases without multiplicity, $T_{2}$ is uniquely fixed by $T_{1}=t_{1}, T_{3}=t_{3}$. In cases with multiplicity, (where the $\rho$-label is needed), the largest of the possible $T_{2}$ values is chosen. Note that eq. (B.1) is a generalization of the $\mathrm{SU}(2)$ phase convention, $\left\langle T_{1} T_{1} T_{2} M_{T_{2}}=\left(T_{3}-T_{1}\right) \mid T_{3} T_{3}\right\rangle>0$.

With the vector coherent state constructions (I) and (II) for particle and hole states, the phase convention (B.1) leads to the very general $1 \leftrightarrow 3$ interchange symmetry relation for the $1 \times 2 \rightarrow 3$ coupling

$$
\begin{aligned}
& \left\langle\left(\omega_{1} t\right)_{1}\left(H_{1}\right)_{1} T_{1} i_{1} ;\left(\omega_{1} t\right)_{2}\left(H_{1}\right)_{2} T_{2} i_{2} \|\left(\omega_{1} t\right)_{3}\left(H_{1}\right)_{3} T_{3} i_{3}\right\rangle_{p} \\
& =(-1)^{t_{1}-t_{3}+T_{3}-T_{1}} \sqrt{\frac{\operatorname{dim}\left(\left(\omega_{1} t\right)_{3}\right)\left(2 T_{1}+1\right)}{\operatorname{dim}\left(\left(\omega_{1} t\right)_{1}\right)\left(2 T_{3}+1\right)}} \\
& \quad \times\left\langle\left(\omega_{1} t\right)_{3}\left(H_{1}\right)_{3} T_{3} i_{3} ;\left(\omega_{1} t\right)_{2}-\left(H_{1}\right)_{2} T_{2} i_{2}^{c} \|\left(\omega_{1} t\right)_{1}\left(H_{1}\right)_{1} T_{1} i_{1}\right\rangle_{p},
\end{aligned}
$$

where the phase factor arises from two sources, a factor $(-1)^{t_{1}+M_{T_{2}}-t_{3}}$ for the full $\operatorname{SO}(5)$ Wigner coefficient, and the factor $(-1)^{T_{1}+M_{T_{2}}-T_{3}}$ which is the $1 \leftrightarrow 3$ interchange phase factor for the conventional $\mathrm{SU}(2)$ Wigner coefficient which is factored out of the full $\mathrm{SO}(5)$ Wigner coefficient to yield the double-barred $\mathrm{SO}(5) \supset \mathrm{U}(2)$ reduced Wigner coefficient. The phase factor $(-1)^{t_{1}+M_{1}-t_{3}}$ arises in the following way. The factor $(-1)^{M_{r_{2}}}$ is required by the standard angular momentum conjugation property. The factor $(-1)^{t_{1}-t_{3}}$ insures that the standard and generalized Condon-Shortley phase convention, (B.1), are satisfied for the states with $M_{T_{1}}=t_{1}, M_{T_{3}}=t_{3}$, and hence $M_{T_{2}}=t_{3}-t_{1}$. The symmetry relation (B.2) has been used throughout the text. It is often needed to cast the $\mathrm{SO}(5) \supset \mathrm{U}(2)$ Wigner coefficients into their simplest possible form.

SO(5) Wigner coefficients involving particle states, with $H_{1} \leqslant 0$, are now to be evaluated with the formulas of tables $4-6$ and the $K$-matrix elements which follow from appendix A. Coefficients involving hole states, with $H_{1} \geqslant 0$, are then to be obtained from these by the particle-hole conjugation symmetry property. For the Wigner coefficients of this investigation involving coupling of an arbitrary irreducible representation $\left(\omega_{1} t\right)$ with the 4 -dimensional representation $\left(\begin{array}{l}1 \\ 22\end{array}\right)$, the 5 -dimensional vector representation (10), and the 10 -dimensional regular representation (11), this symmetry property has the simple form

$$
\begin{aligned}
& \left\langle\left(\omega_{1} t\right)_{1}-\left(H_{1}\right)_{1} T_{1} i_{1} ;\left(\omega_{1} t\right)_{2}-\left(H_{1}\right)_{2} T_{2} i_{2}^{c} \|\left(\omega_{1} t\right)_{3}-\left(H_{1}\right)_{3} T_{3} i_{3}^{c}\right\rangle_{,} \\
& =(-1)^{t_{1}+t_{2}-t_{3}+T_{3}-T_{1}-T_{2}}\left\langle\left(\omega_{1} t\right)_{1}\left(H_{1}\right)_{1} T_{1} i_{1} ;\left(\omega_{1} t\right)_{2}\left(H_{1}\right)_{2} T_{2} i_{2} \|\left(\omega_{1} t\right)_{3}\left(H_{1}\right)_{3} T_{3} i_{3}\right\rangle_{p} .
\end{aligned}
$$


For couplings involving higher-dimensional irreducible representations $\left(\omega_{1} t\right)_{2}$ with more complicated multiplicity possibilities, $\rho$, additional $\rho$-dependent phase factors may come into play.

The phase conventions of the earlier tabulations of refs. ${ }^{9,10}$ ) are unfortunately dependent on the explicit somewhat more cumbersome state constructions of these earlier investigations. The results of the present investigation, however, have been found to agree with the earlier tabulations in all those cases involving only states with 1-dimensional $\left(K K^{\dagger}\right)$ matrices, with the exception of some overall phase factors. Certain columns of the earlier Wigner-coefficient unitary transformation matrices have to be multiplied by factors of $(-1)$ to bring them into agreement with the results of tables 4-6. Once the overall phase has been established, through the evaluation of a simple special case, e.g., the earlier tabulations can be used in conjunction with the present results.

\section{References}

1) D.J. Rowe, J. Math. Phys. 25 (1984) 2662

2) D.J. Rowe, G. Rosensteel and R. Gilmore, J. Math. Phys. 26 (1985) 2787

3) J. Deenen and C. Quesne, J. Math. Phys. 25 (1984) 1638, 2354

4) C. Quesne, J. Math. Phys. 27 (1986) 428, 869

5) D.J. Rowe, R. Le Blanc and K.T. Hecht, J. Math. Phys. 29 (1988) 287

6) K.T. Hecht, The vector coherent state method and its application to problems of higher symmetries, Lecture Notes in Physics 290 (Springer, Berlin, 1987).

7) R. Le Blanc and K.T. Hecht, J. of Phys. A20 (1987) 4613

8) R. Le Blanc and L.C. Biedenharn, J. of Phys. A22 (1989) 31

9) K.T. Hecht and J.P. Elliott, Nucl. Phys. A438 (1985) 29

10) K.T. Hecht, Nucl. Phys. A102 (1967) 11; and references therein

11) R.P. Hemenger and K.T. Hecht, Nucl. Phys. A145 (1970) 468; and references therein 